\title{
Feedback Stabilization of the Two-Dimensional Navier-Stokes Equations by Value Function Approximation
}

\author{
Tobias Breiten ${ }^{1} \cdot$ Karl Kunisch $^{1,2} \cdot$ Laurent Pfeiffer $^{1}$
}

Published online: 18 June 2019

(c) The Author(s) 2019

\begin{abstract}
The value function associated with an optimal control problem subject to the NavierStokes equations in dimension two is analyzed. Its smoothness is established around a steady state, moreover, its derivatives are shown to satisfy a Riccati equation at the order two and generalized Lyapunov equations at the higher orders. An approximation of the optimal feedback law is then derived from the Taylor expansion of the value function. A convergence rate for the resulting controls and closed-loop systems is demonstrated.
\end{abstract}

Keywords Stabilization - 2-D Navier-Stokes equations · Value function · Taylor expansion $\cdot$ Feedback control

Mathematics Subject Classification 35Q35 - 49J20 - 49N35 - 93D05 · 93D15

\section{Introduction}

In this work we continue our investigations of the value function associated with infinite-horizon optimal control problems of partial differential equations, that we initiated in $[15,17]$. We consider a stabilization problem of the Navier-Stokes equations in dimension two and focus on the regularity of the value function and its characterization as a solution to a Hamilton-Jacobi-Bellman (HJB) equation. This task has been the subject of tremendous research, for optimal control problems of a general

Karl Kunisch

karl.kunisch@uni-graz.at

Tobias Breiten

tobias.breiten@uni-graz.at

Laurent Pfeiffer

laurent.pfeiffer@uni-graz.at

1 Institute of Mathematics, University of Graz, Graz, Austria

2 RICAM Institute, Austrian Academy of Sciences, Linz, Austria 
structure, in general associated with finite-dimensional dynamical systems. The use of the notion of viscosity solutions has allowed to deal with the low regularity of the value function. In the present paper, to the contrary, we show that the value function is smooth and that the HJB equation is satisfied in the strict sense, in a neighborhood of the steady state. Moreover, we show that the derivatives of the value function, at the steady state, are solutions to an algebraic Riccati equation (for the order 2) and to linear equations, called generalized Lyapunov equations, for the higher orders. The main interest of these results is the fact that polynomial feedback laws can be derived from Taylor approximations of the value function. Moreover their efficiency can be analyzed.

From a methodological point of view, we mainly follow the techniques that we laid out for bilinear optimal control problems (such as control problems of the Fokker-Planck equation) in [15,17]. The Navier-Stokes control system considered here requires a different functional analytic treatment. In fact, the involved nonlinear terms must be tackled with different estimates, to guarantee, for example, the wellposedness of the closed-loop system. They also lead to different generalized Lyapunov equations. Moreover, from the point of view of open-loop control of the Navier-Stokes equation, this paper contains results on infinite-horizon optimal control which are not readily available elsewhere.

Feedback stabilization of the Navier-Stokes equations has been and still is an active topic of research. Among the numerous works, we refer to, e.g., [6,7,10,24,38], and the references therein. For literature concerning open-loop optimal control of the NavierStokes equations, we can only cite a small selection [13,18,19,21,22,27,30,42].

The technique of approximation of the value function with a Taylor expansion dates back to $[3,35]$, where optimal control problems associated to finite-dimensional control systems were investigated. We also quote follow-up work, for instance in $[2,8,36]$. For infinite-dimensional problems, we are only aware of [15,17]. In [16], the numerical solvability of the Lyapunov equations has been addressed. Model reduction techniques based on balanced truncation have been used in this reference to cope with the curse of dimensionality encountered when dealing with PDE controlled systems.

Let us next specify the problem which will be investigated in this paper. Throughout $\Omega \subset \mathbb{R}^{2}$ denotes a bounded domain with Lipschitz boundary $\Gamma$. Given two vector valued functions $\varphi$ and $\psi$, we consider a solution $(\overline{\mathbf{z}}, \bar{q})$ of the stationary NavierStokes equations

$$
\begin{aligned}
-v \Delta \overline{\mathbf{z}}+(\overline{\mathbf{z}} \cdot \nabla) \overline{\mathbf{z}}+\nabla \bar{q} & =\varphi & & \text { in } \Omega, \\
\operatorname{div} \overline{\mathbf{z}} & =0 & & \text { in } \Omega, \\
\overline{\mathbf{z}} & =\psi & & \text { on } \Gamma .
\end{aligned}
$$

Our goal is to find a control $u$ such that the solution $(\mathbf{z}, q)$ to the transient Navier-Stokes equations 


$$
\begin{aligned}
\frac{\partial \mathbf{z}}{\partial t} & =v \Delta \mathbf{z}-(\mathbf{z} \cdot \nabla) \mathbf{z}-\nabla q+\boldsymbol{\varphi}+\tilde{B} u & & \text { in } \Omega \times(0, T), \\
\operatorname{div} \mathbf{z} & =0 & & \text { in } \Omega \times(0, T), \\
\mathbf{z} & =\psi & & \text { on } \Gamma \times(0, T), \\
\mathbf{z}(0) & =\overline{\mathbf{z}}+\mathbf{y}_{0} & &
\end{aligned}
$$

is stabilized around $\overline{\mathbf{z}}$, i.e., $\lim _{t \rightarrow \infty} \mathbf{z}(t)=\overline{\mathbf{z}}$ provided the initial perturbation $\mathbf{y}_{0}$ is small in an appropriate sense. The control operator $\tilde{B}$ will be defined below. Throughout this work, we assume that $\operatorname{div} \mathbf{y}_{0}=0$. Our results are concerned with feedback stabilization of (2) and for this purpose, we consider new state variables $(\mathbf{y}, p):=(\mathbf{z}, q)-(\overline{\mathbf{z}}, \bar{q})$ which satisfy the following generalized Navier-Stokes equations

$$
\begin{aligned}
\frac{\partial \mathbf{y}}{\partial t} & =v \Delta \mathbf{y}-(\mathbf{y} \cdot \nabla) \overline{\mathbf{z}}-(\overline{\mathbf{z}} \cdot \nabla) \mathbf{y}-(\mathbf{y} \cdot \nabla) \mathbf{y}-\nabla p+\tilde{B} u & & \text { in } \Omega \times(0, T), \\
\operatorname{div} \mathbf{y} & =0 & & \text { in } \Omega \times(0, T), \\
\mathbf{y} & =0 & & \text { on } \Gamma \times(0, T), \\
\mathbf{y}(0) & =\mathbf{y}_{0} . & &
\end{aligned}
$$

The following sections are structured as follows. The problem statement and fundamental results on the state-equation on the time interval $[0, \infty)$ are given in Sect. 2. Section 3 contains the existence theory of optimal controls, the adjoint equation, sensitivity analysis, and differentiability of the value function. The characterization of all higher order derivatives of the value as solutions to generalized Lyapunov equations are provided in Sect. 4. Section 5 contains the Taylor expansion of the value function, and estimates for convergence rates between the optimal solution and its approximation on the basis of feedback solutions obtained from derivatives of the value function. The paper closes with a very short outlook.

Notation For Hilbert spaces $V \subset Y$ with dense and compact embedding, we consider the Gelfand triple $V \subset Y \subset V^{\prime}$ where $V^{\prime}$ denotes the topological dual of $V$ with respect to the pivot space $Y$. Given $T \in \mathbb{R}$ we consider the space

$$
W(0, T)=\left\{y \in L^{2}(0, T ; V) \mid \frac{\mathrm{d}}{\mathrm{d} t} y \in L^{2}\left(0, T ; V^{\prime}\right)\right\} .
$$

For $T=\infty$, the space $W(0, T)$ will be denoted by $W_{\infty}$. For vector-valued functions $\mathbf{f} \in\left(L^{2}(\Omega)\right)^{2}$, we use the notation $\mathbf{f} \in \mathbb{L}^{2}(\Omega)$. Elements $\mathbf{f} \in \mathbb{L}^{2}(\Omega)$ will be denoted in boldface and are distinguished from real-valued functions $g \in L^{2}(\Omega)$. Similarly, we use $\mathbb{H}^{2}(\Omega)$ for the space $\left(H^{2}(\Omega)\right)^{2}$ and $\mathbb{H}_{0}^{1}(\Omega)$ for $\left(H_{0}^{1}(\Omega)\right)^{2}$. Given a closed, densely defined linear operator $(A, \mathcal{D}(A))$ in $Y$, its adjoint (again considered as an operator in $Y)$ will be denoted with $\left(A^{*}, \mathcal{D}\left(A^{*}\right)\right)$.

Let us introduce some notation that will be needed for the description of polynomial mappings. For $\delta \geq 0$ and a Hilbert space $Y$, we denote by $B_{Y}(\delta)$ the closed ball in $Y$ 
with radius $\delta$ and center 0 . For $k \geq 1$, we make use of the following norm:

$$
\left\|\left(y_{1}, \ldots, y_{k}\right)\right\|_{Y^{k}}=\max _{i=1, \ldots, k}\left\|y_{i}\right\|_{Y} .
$$

Given a Hilbert space $Z$, we say that $\mathcal{T}: Y^{k} \rightarrow Z$ is a bounded multilinear mapping (or bounded multilinear form when $Z=\mathbb{R}$ ) if for all $i \in\{1, \ldots, k\}$ and for all $\left(z_{1}, \ldots, z_{i-1}, z_{i+1}, \ldots, z_{k}\right) \in Y^{k-1}$, the mapping $z \in Y \mapsto$ $\mathcal{T}\left(z_{1}, \ldots, z_{i-1}, z, z_{i+1}, \ldots, z_{k}\right) \in Z$ is linear and

$$
\|\mathcal{T}\|:=\sup _{y \in B_{Y^{k}}(1)}\|\mathcal{T}(y)\|_{Z}<\infty
$$

The set of bounded multilinear mappings on $Y^{k}$ will be denoted by $\mathcal{M}\left(Y^{k}, Z\right)$. For all $\mathcal{T} \in \mathcal{M}\left(Y^{k}, Z\right)$ and for all $\left(z_{1}, \ldots, z_{k}\right) \in Y^{k}$,

$$
\left\|\mathcal{T}\left(z_{1}, \ldots, z_{k}\right)\right\|_{Z} \leq\|\mathcal{T}\| \prod_{i=1}^{k}\left\|z_{i}\right\|_{Y}
$$

Given a bounded multilinear form $\mathcal{T}$ and $z_{2}, \ldots, z_{k} \in Y^{k-1}$, we denote by $\mathcal{T}\left(\cdot, z_{2}, \ldots, z_{k}\right)$ the bounded linear form $z_{1} \in Y \mapsto \mathcal{T}\left(z_{1}, \ldots, z_{k}\right) \in \mathbb{R}$. It will be very often identified with its Riesz representative. Note that

$$
\left\|\mathcal{T}\left(\cdot, z_{2}, \ldots, z_{k}\right)\right\|_{Y}=\sup _{z_{1} \in B_{Y}(1)} \mathcal{T}\left(z_{1}, \ldots, z_{k}\right) \leq\|\mathcal{T}\| \prod_{i=2}^{k}\left\|z_{i}\right\|_{Y}
$$

Bounded multilinear mappings $\mathcal{T} \in \mathcal{M}\left(Y^{k}, Z\right)$ are said to be symmetric if for all $z_{1}, \ldots, z_{k} \in Y^{k}$ and for all permutations $\sigma$ of $\{1, \ldots, k\}$,

$$
\mathcal{T}\left(z_{\sigma(1)}, \ldots, z_{\sigma(k)}\right)=\mathcal{T}\left(z_{1}, \ldots, z_{k}\right) .
$$

Finally, given two multilinears mappings $\mathcal{T}_{1} \in \mathcal{M}\left(Y^{k}, Z\right)$ and $\mathcal{T}_{2} \in \mathcal{M}\left(Y^{\ell}, Z\right)$, we denote by $\mathcal{T}_{1} \otimes \mathcal{T}_{2}$ the bounded multilinear form defined by

$$
\mathcal{T}_{1} \otimes \mathcal{T}_{2}\left(z_{1}, \ldots, z_{k+\ell}\right)=\left\langle\mathcal{T}_{1}\left(z_{1}, \ldots, z_{k}\right), \mathcal{T}_{2}\left(z_{k+1}, \ldots, z_{k+\ell}\right)\right\rangle_{Z}
$$

Throughout the manuscript, we use $M$ as a generic constant that might change its value between consecutive lines.

\section{Problem Formulation}

\subsection{Abstract Cauchy Problem}

In this section, we formulate system (3) as an abstract Cauchy problem on a suitable Hilbert space and, subsequently, define the stabilization problem of interest. This 
procedure is quite standard, see, for instance, $[6,7,24,38,40]$ for details. We introduce the spaces

$$
\begin{aligned}
& Y:=\left\{\mathbf{y} \in \mathbb{L}^{2}(\Omega) \mid \operatorname{div} \mathbf{y}=0, \mathbf{y} \cdot \vec{n}=0 \text { on } \Gamma\right\} \\
& V:=\left\{\mathbf{y} \in \mathbb{H}_{0}^{1}(\Omega) \mid \operatorname{div} \mathbf{y}=0\right\}
\end{aligned}
$$

It is well-known that $Y$ is a closed subspace of $\mathbb{L}^{2}(\Omega)$. Moreover, we have the orthogonal decomposition

$$
\mathbb{L}^{2}(\Omega)=Y \oplus Y^{\perp},
$$

where

$$
Y^{\perp}=\left\{\mathbf{z}=\nabla p \mid p \in H^{1}(\Omega)\right\}
$$

see, e.g., [40, p. 15]. By $P$ we denote the Leray projector $P: \mathbb{L}^{2}(\Omega) \rightarrow Y$ which is the orthogonal projector in $\mathbb{L}^{2}(\Omega)$ onto $Y$. Following, e.g., [6], we define a trilinear form $s$ by

$$
s(\mathbf{u}, \mathbf{v}, \mathbf{w}):=\int_{\Omega} \sum_{i, j=1}^{2} u_{i} w_{j} \frac{\partial v_{j}}{\partial x_{i}} \mathrm{~d} x=\langle(\mathbf{u} \cdot \nabla) \mathbf{v}, \mathbf{w}\rangle_{\mathbb{L}^{2}(\Omega)}, \quad \forall \mathbf{u}, \mathbf{v}, \mathbf{w} \in V
$$

and a nonlinear operator $F: V \rightarrow V^{\prime}$ by

$$
\langle F(\mathbf{y}), \mathbf{w}\rangle_{V^{\prime}, V}:=s(\mathbf{y}, \mathbf{y}, \mathbf{w}), \quad \forall \mathbf{w} \in V
$$

For the bilinear mapping associated with the linearization of $F$, we introduce the operator

$$
N: V \times V \rightarrow V^{\prime},\langle N(\mathbf{y}, \mathbf{z}), \mathbf{w}\rangle_{V^{\prime}, V}:=s(\mathbf{y}, \mathbf{z}, \mathbf{w})
$$

The Oseen-Operator is then defined by

$$
A_{0}: V \times V \rightarrow V^{\prime},\left\langle A_{0}(\mathbf{y}, \mathbf{z}), \mathbf{w}\right\rangle_{V^{\prime}, V}:=\langle N(\mathbf{y}, \mathbf{z})+N(\mathbf{z}, \mathbf{y}), \mathbf{w}\rangle_{V^{\prime}, V}
$$

The following well-known results (see, e.g., [6], [40, Lemma III.3.4]) concerning $s$ and $N$ will be used frequently throughout the paper.

Proposition 1 The following properties hold for $N$ and $s$ :

(i) $\|N(\mathbf{y}, \mathbf{z})\|_{V^{\prime}} \leq M\|\mathbf{y}\|_{Y}^{\frac{1}{2}}\|\mathbf{z}\|_{Y}^{\frac{1}{2}}\|\mathbf{y}\|_{V}^{\frac{1}{2}}\|\mathbf{z}\|_{V}^{\frac{1}{2}}$, for all $\mathbf{y}, \mathbf{z} \in V$,

(ii) $s(\mathbf{y}, \mathbf{z}, \mathbf{w})=-s(\mathbf{y}, \mathbf{w}, \mathbf{z})$, for all $\mathbf{y}, \mathbf{z}, \mathbf{w} \in V$.

With the previous result, we obtain similar properties for time-varying functions $\mathbf{y}, \mathbf{z}, \mathbf{w}$. 
Lemma 2 Let $T \in(0, \infty]$. For all $\mathbf{y} \in W(0, T)$, for all $\mathbf{z} \in W(0, T)$, and for all $\mathbf{w} \in L^{2}(0, T ; V)$,

$$
\begin{aligned}
& \langle N(\mathbf{y}, \mathbf{z}), \mathbf{w}\rangle_{L^{2}\left(0, T ; V^{\prime}\right), L^{2}(0, T ; V)} \\
& \quad \leq M\|\mathbf{y}\|_{L^{\infty}(0, T ; Y)}^{\frac{1}{2}}\|\mathbf{y}\|_{L^{2}(0, T ; V)}^{\frac{1}{2}}\|\mathbf{z}\|_{L^{\infty}(0, T ; Y)}^{\frac{1}{2}}\|\mathbf{z}\|_{L^{2}(0, T ; V)}^{\frac{1}{2}}\|\mathbf{w}\|_{L^{2}(0, T ; V)} .
\end{aligned}
$$

Moreover, if $\mathbf{w} \in L^{\infty}(0, T ; V)$,

$$
\begin{aligned}
& \langle N(\mathbf{y}, \mathbf{z}), \mathbf{w}\rangle_{L^{2}\left(0, T ; V^{\prime}\right), L^{2}(0, T ; V)} \\
& \quad \leq M\|\mathbf{y}\|_{L^{2}(0, T ; Y)}^{\frac{1}{2}}\|\mathbf{y}\|_{L^{2}(0, T ; V)}^{\frac{1}{2}}\|\mathbf{z}\|_{L^{2}(0, T ; Y)}^{\frac{1}{2}}\|\mathbf{z}\|_{L^{2}(0, T ; V)}^{\frac{1}{2}}\|\mathbf{w}\|_{L^{\infty}(0, T ; V)},
\end{aligned}
$$

where $M$ is the constant given by Proposition 1.

Proof Using Proposition 1 and Cauchy-Schwarz inequality (two times), we obtain that

$$
\begin{aligned}
& \langle N(\mathbf{y}, \mathbf{z}), \mathbf{w}\rangle_{L^{2}\left(0, T ; V^{\prime}\right), L^{2}(0, T ; V)} \leq M \int_{0}^{T}\|\mathbf{y}(t)\|_{Y}^{\frac{1}{2}}\|\mathbf{y}(t)\|_{V}^{\frac{1}{2}}\|\mathbf{z}(t)\|_{Y}^{\frac{1}{2}}\|\mathbf{z}(t)\|_{V}^{\frac{1}{2}}\|\mathbf{w}(t)\|_{V} \mathrm{~d} t \\
& \quad \leq M\|\mathbf{y}\|_{L^{2}(0, T ; V)}^{\frac{1}{2}}\|\mathbf{z}\|_{L^{2}(0, T ; V)}^{\frac{1}{2}}\left(\int_{0}^{T}\|\mathbf{y}(t)\|_{Y}\|\mathbf{z}(t)\|_{Y}\|\mathbf{w}(t)\|_{V}^{2}\right)^{\frac{1}{2}}
\end{aligned}
$$

The two inequalities easily follow.

Corollary 3 There exists $M>0$ such that for all $\mathbf{y}$ and $\mathbf{z} \in W_{\infty}$,

$$
\|N(\mathbf{y}, \mathbf{z})\|_{L^{2}\left(0, \infty ; V^{\prime}\right)} \leq M\|\mathbf{y}\|_{W_{\infty}}\|\mathbf{z}\|_{W_{\infty}}
$$

For $\overline{\mathbf{z}} \in V$, we further introduce the Stokes-Oseen operator $A$ via

$$
\mathcal{D}(A)=\mathbb{H}^{2}(\Omega) \cap V, \quad A \mathbf{y}=P(v \Delta \mathbf{y}-(\mathbf{y} \cdot \nabla) \overline{\mathbf{z}}-(\overline{\mathbf{z}} \cdot \nabla) \mathbf{y}) .
$$

Considered as operator in $\mathbb{L}^{2}(\Omega)$ the adjoint $A^{*}$, as operator in $\mathbb{L}^{2}(\Omega)$, can be characterized by (see, e.g., [38])

$$
\mathcal{D}\left(A^{*}\right)=\mathbb{H}^{2}(\Omega) \cap V, \quad A^{*} \mathbf{p}=P\left(\nu \Delta \mathbf{p}-(\nabla \overline{\mathbf{z}})^{T} \mathbf{p}+(\overline{\mathbf{z}} \cdot \nabla) \mathbf{p}\right) .
$$

We note that as a consequence of Proposition 1, the operator $A$ can be extended to a bounded linear operator from $V$ to $V^{\prime}$ in the following manner:

$$
\langle A \mathbf{y}, \mathbf{w}\rangle_{V^{\prime}, V}=-v\langle\nabla \mathbf{y}, \nabla \mathbf{w}\rangle_{\mathbb{L}^{2}(\Omega)}-\left\langle A_{0}(\overline{\mathbf{z}}, \mathbf{y}), \mathbf{w}\right\rangle_{V^{\prime}, V}
$$

Note that this extension is consistent, since by definition of the Leray projector $P$, we have $\langle P \mathbf{y}, \mathbf{w}\rangle_{Y}=\langle\mathbf{y}, \mathbf{w}\rangle_{Y}$ for all $\mathbf{y} \in \mathbb{L}^{2}(\Omega)$ and for all $\mathbf{w} \in V$. Similarly, $A^{*}$ can be extended to a bounded linear operator from $V$ to $V^{\prime}$. 
The control operator is chosen to satisfy $\tilde{B} \in \mathcal{L}\left(U, \mathbb{L}^{2}(\Omega)\right)$. We further define $B:=P \tilde{B} \in \mathcal{L}(U, Y)$. The controlled state Eq. (3) can now be formulated as the abstract control system

$$
\dot{\mathbf{y}}(t)=A \mathbf{y}-F(\mathbf{y})+B u, \quad \mathbf{y}(0)=\mathbf{y}_{0},
$$

where the pressure $p$ is eliminated. We can finally formulate the stabilization problem as an infinite-horizon optimal control problem:

$$
\inf _{\substack{\mathbf{y} \in W_{\infty} \\ u \in L^{2}(0, \infty ; U)}} J(\mathbf{y}, u), \quad \text { subject to: } e(\mathbf{y}, u)=\left(0, \mathbf{y}_{0}\right),
$$

where $J: W_{\infty} \times L^{2}(0, \infty ; U) \rightarrow \mathbb{R}$ and $e: W_{\infty} \times L^{2}(0, \infty ; U) \rightarrow L^{2}\left(0, \infty ; V^{\prime}\right) \times Y$ are defined by

$$
\begin{aligned}
& J(\mathbf{y}, u)=\frac{1}{2} \int_{0}^{\infty}\|\mathbf{y}\|_{Y}^{2} \mathrm{~d} t+\frac{\alpha}{2} \int_{0}^{\infty}\|u(t)\|_{U}^{2} \mathrm{~d} t \\
& e(\mathbf{y}, u)=(\dot{\mathbf{y}}-(A \mathbf{y}-F(\mathbf{y})+B u), \mathbf{y}(0)) .
\end{aligned}
$$

Let us note that $e: W_{\infty} \times L^{2}(0, \infty ; U) \rightarrow L^{2}\left(0, \infty ; V^{\prime}\right) \times Y$ is well-defined by Corollary 3.

\subsection{Assumptions and First Properties}

Throughout the article we assume that the following assumptions hold true.

Assumption A1 The stationary solution satisfies $\overline{\mathbf{z}} \in V$.

Assumption A2 There exists an operator $K \in \mathcal{L}(Y, U)$ such that the semigroup $e^{(A-B K) t}$ is exponentially stable on $Y$.

Assumption A2 concerning the exponential feedback stabilizability of the StokesOseen operator is well investigated. We refer e.g. to [6] where finite-dimensional feedback operators are constructed on the basis of spectral decomposition or alternatively by Riccati theory. In this case A2 can be satisfied with $U=\mathbb{R}^{m}$, for $m$ appropriately large. Alternatively, we can rely on exact controllability results as obtained in [23]. They imply that the finite cost criterion holds. We can then rely on classical results, see, e.g., [37] which guarantee the existence of a stabilizing feedback operator.

Let us discuss some important consequences of the above definitions and assumptions.

Consequence $\mathrm{C} 1$ There exists $\lambda \geq 0$ and $\theta>0$ such that

$$
\langle(\lambda I-A) \mathbf{v}, \mathbf{v}\rangle_{Y} \geq \theta\|\mathbf{v}\|_{V}^{2}, \quad \text { for all } \mathbf{v} \in V
$$


Hence, A generates an analytic semigroup $e^{A t}$ on $Y$, see [12, Part II, Chapter 1, Theorem 2.12].

Consequence C2 For all $\mathbf{y}_{0} \in Y$, for all $\mathbf{f} \in L^{2}\left(0, \infty ; V^{\prime}\right)$, and for all $T>0$, there exists a unique solution $\mathbf{y} \in W(0, T)$ to the system

$$
\dot{\mathbf{y}}=A \mathbf{y}+\mathbf{f}, \quad \mathbf{y}(0)=\mathbf{y}_{0} .
$$

This solution satisfies

$$
\|\mathbf{y}\|_{W(0, T)} \leq c(T)\left(\left\|\mathbf{y}_{0}\right\|_{Y}+\|\mathbf{f}\|_{L^{2}\left(0, \infty ; V^{\prime}\right)}\right)
$$

with a continuous function $c$. Assuming that $\mathbf{y} \in L^{2}(0, \infty ; Y)$, we consider the equivalent equation

$$
\dot{\mathbf{y}}=\underbrace{(A-\lambda I)}_{A_{\lambda}} \mathbf{y}+\underbrace{\lambda \mathbf{y}+\mathbf{f}}_{\mathbf{f}_{\lambda}}, \quad \mathbf{y}(0)=\mathbf{y}_{0},
$$

where $\mathbf{f}_{\lambda} \in L^{2}\left(0, \infty ; V^{\prime}\right)$. By (18), the operator $A_{\lambda}$ generates an analytic, exponentially stable, semigroup on $Y$ satisfying $\left\|e^{A_{\lambda} t}\right\|_{Y} \leq e^{-\delta t}$ for some $\delta>0$ independent of $t \geq 0$, see [12, Theorem II.1.2.12]. It follows that $\mathbf{y} \in W_{\infty}$ and there exists $M_{\lambda}$ such that with

$$
\|\mathbf{y}\|_{W_{\infty}} \leq M_{\lambda}\left(\left\|\mathbf{y}_{0}\right\|_{Y}+\left\|\mathbf{f}_{\lambda}\right\|_{L^{2}\left(0, \infty ; V^{\prime}\right)}\right)
$$

This estimate is obtained by adapting [12, Corollary II.3.2.1] and [12, Theorem II.3.2.2] from the temporal domain $(0, T)$ to $(0, \infty)$, which can be achieved using the exponential stability of $e^{A_{\lambda} t}$.

Lemma 4 There exists a constant $C>0$ such that for all $\delta \in[0,1]$ and for all $\mathbf{y}$ and $\mathbf{z} \in W_{\infty}$ with $\|\mathbf{y}\|_{W_{\infty}} \leq \delta$ and $\|\mathbf{z}\|_{W_{\infty}} \leq \delta$, it holds that

$$
\|F(\mathbf{y})-F(\mathbf{z})\|_{L^{2}\left(0, \infty ; V^{\prime}\right)} \leq \delta C\|\mathbf{y}-\mathbf{z}\|_{W_{\infty}} .
$$

Proof We have

$$
\begin{aligned}
\|F(\mathbf{y})-F(\mathbf{z})\|_{L^{2}\left(0, \infty ; V^{\prime}\right)} & =\|N(\mathbf{y}, \mathbf{y})-N(\mathbf{z}, \mathbf{z})\|_{L^{2}\left(0, \infty ; V^{\prime}\right)} \\
& \leq\|N(\mathbf{y}-\mathbf{z}, \mathbf{y})\|_{L^{2}\left(0, \infty ; V^{\prime}\right)}+\|N(\mathbf{z}, \mathbf{y}-\mathbf{z})\|_{L^{2}\left(0, \infty ; V^{\prime}\right)} .
\end{aligned}
$$

The assertion now easily follows from Corollary 3.

The following lemma is formulated for an abstract generator $A_{s}$ of an analytic semigroup on $Y$. It will subsequently be used to address the asymptotic behavior of the nonlinear system (15). We point out that the statement is similar to [38, Theorem 6.1] which, since it addresses the boundary control case, assumes a slightly more regular initial condition $\mathbf{y}_{0} \in \mathbb{H}^{\varepsilon}(\Omega) \cap Y$. 
Lemma 5 Let $A_{s}$ be the generator of an exponentially stable analytic semigroup $e^{A_{s} t}$ on $Y$ such that (18) holds. Let $C$ denote the constant from Lemma 4. Then there exists a constant $M_{S}$ such that for all $\mathbf{y}_{0} \in Y$ and $\mathbf{f} \in L^{2}\left(0, \infty ; V^{\prime}\right)$ with

$$
\gamma:=\left\|\mathbf{y}_{0}\right\|_{Y}+\|\mathbf{f}\|_{L^{2}\left(0, \infty ; V^{\prime}\right)} \leq \frac{1}{4 C M_{S}^{2}}
$$

the system

$$
\dot{\mathbf{y}}=A_{s} \mathbf{y}-F(\mathbf{y})+\mathbf{f}, \quad \mathbf{y}(0)=\mathbf{y}_{0}
$$

has a unique solution $\mathbf{y}$ in $W_{\infty}$, which moreover satisfies

$$
\|\mathbf{y}\|_{W_{\infty}} \leq 2 M_{s} \gamma
$$

Proof We follow the line of argumentation provided in the proof [38, Theorem 6.1]. Since the semigroup $e^{A_{s} t}$ is exponentially stable on $Y$, it follows that for all $\left(\mathbf{y}_{0}, \mathbf{g}\right) \in$ $Y \times L^{2}\left(0, \infty ; V^{\prime}\right)$ the system

$$
\dot{\mathbf{z}}=A_{s} \mathbf{z}+\mathbf{g}, \quad \mathbf{z}(0)=\mathbf{y}_{0}
$$

has a unique solution $\mathbf{z} \in W_{\infty}$. Moreover, there exists a constant $M_{S}$ such that

$$
\|\mathbf{z}\|_{W_{\infty}} \leq M_{S}\left(\left\|\mathbf{y}_{0}\right\|_{Y}+\|\mathbf{g}\|_{L^{2}\left(0, \infty ; V^{\prime}\right)}\right)
$$

Without loss of generality we can assume that $M_{S} \geq \frac{1}{2 C}$. We claim that the constant $M_{s}$ is the one announced in the assertion. This will be shown by a fixedpoint argument applied to the system (20). For this purpose, let us define $\mathcal{M}=$ $\left\{\mathbf{y} \in W_{\infty} \mid\|\mathbf{y}\|_{W_{\infty}} \leq 2 M_{s} \gamma\right\}$ and let us define the mapping $\mathcal{Z}: \mathcal{M} \ni \mathbf{y} \mapsto \mathbf{z}=$ $\mathcal{Z}(\mathbf{y}) \in W_{\infty}$, where $\mathbf{z}$ is the unique solution of

$$
\dot{\mathbf{z}}=A_{s} \mathbf{z}-F(\mathbf{y})+\mathbf{f}, \quad \mathbf{z}(0)=\mathbf{y}_{0} .
$$

If there exists a unique fixed point of $\mathcal{Z}$, then it is a unique solution of (20) in $\mathcal{M}$. With $C$ and $M_{s}$ given, we shall use Lemma 4 with $\delta=2 M_{s} \gamma \leq \frac{1}{2 C M_{s}} \leq 1$. Together with (21), it follows that

$$
\begin{aligned}
\|\mathbf{z}\|_{W_{\infty}} & \leq M_{S}\left(\|F(\mathbf{y})\|_{L^{2}\left(0, \infty ; V^{\prime}\right)}+\|\mathbf{f}\|_{L^{2}\left(0, \infty ; V^{\prime}\right)}+\left\|\mathbf{y}_{0}\right\|_{Y}\right) \\
& \leq M_{S}\left(\frac{1}{2 M_{S}}\|\mathbf{y}\|_{W_{\infty}}+\gamma\right) \leq 2 M_{s} \gamma
\end{aligned}
$$

This implies $\mathcal{Z}(\mathcal{M}) \subseteq \mathcal{M}$. For $\mathbf{y}_{1}, \mathbf{y}_{2} \in \mathcal{M}$ consider now $\mathbf{z}=\mathcal{Z}\left(\mathbf{y}_{1}\right)-\mathcal{Z}\left(\mathbf{y}_{2}\right)$ solving

$$
\dot{\mathbf{z}}=A_{s} \mathbf{z}-F\left(\mathbf{y}_{1}\right)+F\left(\mathbf{y}_{2}\right), \quad \mathbf{z}(0)=0 .
$$


Again by (21) and Lemma 4 we obtain

$$
\begin{aligned}
\left\|\mathcal{Z}\left(\mathbf{y}_{1}\right)-\mathcal{Z}\left(\mathbf{y}_{2}\right)\right\|_{W_{\infty}} & =\|\mathbf{z}\|_{W_{\infty}} \leq M_{S}\left(\left\|F\left(\mathbf{y}_{1}\right)-F\left(\mathbf{y}_{2}\right)\right\|_{L^{2}\left(0, \infty ; V^{\prime}\right)}\right) \\
& \leq M_{S} \delta C\left\|\mathbf{y}_{1}-\mathbf{y}_{2}\right\|_{W_{\infty}} \leq \frac{1}{2}\left\|\mathbf{y}_{1}-\mathbf{y}_{2}\right\|_{W_{\infty}} .
\end{aligned}
$$

In other words, $\mathcal{Z}$ is a contraction in $\mathcal{M}$ and therefore, there exists a unique $\mathbf{y} \in \mathcal{M}$ such that $\mathcal{Z}(\mathbf{y})=\mathbf{y}$. Regarding uniqueness in $W_{\infty}$, consider two solutions $\mathbf{y}, \mathbf{z} \in W_{\infty}$. For the difference $\mathbf{e}:=\mathbf{y}-\mathbf{z}$ it then holds

$$
\dot{\mathbf{e}}=A_{s} \mathbf{e}-F(\mathbf{y})+F(\mathbf{z}), \quad \mathbf{e}(0)=0 .
$$

Multiplying with e and taking inner products yields

$$
\frac{1}{2} \frac{\mathrm{d}}{\mathrm{d} t}\|\mathbf{e}\|_{Y}^{2}=\left\langle A_{S} \mathbf{e}, \mathbf{e}\right\rangle_{Y}-\langle F(\mathbf{y})-F(\mathbf{z}), \mathbf{e}\rangle_{V^{\prime}, V}
$$

Since $A_{s}$ satisfies an inequality of the form (18), we have

$$
\frac{1}{2} \frac{\mathrm{d}}{\mathrm{d} t}\|\mathbf{e}\|_{Y}^{2} \leq \alpha\|\mathbf{e}\|_{Y}^{2}-\beta\|\mathbf{e}\|_{V}^{2}+\|F(\mathbf{y})-F(\mathbf{z})\|_{V^{\prime}}\|\mathbf{e}\|_{V}
$$

where $\alpha \geq 0$ and $\beta>0$. Using Proposition 1 and Young's inequality we further obtain

$$
\begin{aligned}
\frac{1}{2} \frac{\mathrm{d}}{\mathrm{d} t}\|\mathbf{e}\|_{Y}^{2} \leq & \alpha\|\mathbf{e}\|_{Y}^{2}-\beta\|\mathbf{e}\|_{V}^{2}+M\left(\|\mathbf{e}\|_{Y}^{\frac{1}{2}}\|\mathbf{y}\|_{Y}^{\frac{1}{2}}\|\mathbf{e}\|_{V}^{\frac{1}{2}}\|\mathbf{y}\|_{V}^{\frac{1}{2}}\right. \\
& \left.+\|\mathbf{e}\|_{Y}^{\frac{1}{2}}\|\mathbf{z}\|_{Y}^{\frac{1}{2}}\|\mathbf{e}\|_{V}^{\frac{1}{2}}\|\mathbf{z}\|_{V}^{\frac{1}{2}}\right)\|\mathbf{e}\|_{V} \\
\leq & \alpha\|\mathbf{e}\|_{Y}^{2}-\beta\|\mathbf{e}\|_{V}^{2}+\frac{M}{\iota}\|\mathbf{e}\|_{V}^{2}+\frac{M \iota}{2}\|\mathbf{e}\|_{V}\left(\|\mathbf{e}\|_{Y}\|\mathbf{y}\|_{Y}\left\|_{\mathbf{y}}\right\|_{V}\right. \\
& \left.+\|\mathbf{e}\|_{Y}\|\mathbf{z}\|_{Y}\|\mathbf{z}\|_{V}\right) \\
\leq & \alpha\|\mathbf{e}\|_{Y}^{2}-\beta\|\mathbf{e}\|_{V}^{2}+\frac{M}{\iota}\|\mathbf{e}\|_{V}^{2}+\frac{M \iota}{2 \kappa}\|\mathbf{e}\|_{V}^{2}+\frac{M \iota \kappa}{4}\left(\|\mathbf{e}\|_{Y}^{2}\|\mathbf{y}\|_{Y}^{2}\|\mathbf{y}\|_{V}^{2}\right. \\
& \left.+\|\mathbf{e}\|_{Y}^{2}\|\mathbf{z}\|_{Y}^{2}\|\mathbf{z}\|_{V}^{2}\right) .
\end{aligned}
$$

Taking $\iota$ and $\kappa$ sufficiently large, it holds that

$$
\frac{1}{2} \frac{\mathrm{d}}{\mathrm{d} t}\|\mathbf{e}\|_{Y}^{2} \leq\left(\alpha+\frac{M \iota \kappa}{4}\left(\|\mathbf{y}\|_{Y}^{2}\|\mathbf{y}\|_{V}^{2}+\|\mathbf{z}\|_{Y}^{2}\|\mathbf{z}\|_{V}^{2}\right)\right)\|\mathbf{e}\|_{Y}^{2} .
$$

Since $\mathbf{y}, \mathbf{z} \in W_{\infty}$ and $\mathbf{e}(0)=0$, with Gronwall's inequality, we conclude that $\mathbf{e}(t)=0$ for all $t \geq 0$. Hence, $\mathbf{y}=\mathbf{z}$ showing the uniqueness of the solution in $W_{\infty}$.

The following two corollaries are consequences of Lemmas 4 and 5. The constant $C$ which is employed is the one given by Lemma 4 . 
Corollary 6 There exists a constant $M_{K}>0$ such that for all $\mathbf{y}_{0} \in Y$ and for all $\mathbf{f} \in L^{2}\left(0, \infty ; V^{\prime}\right)$ with

$$
\gamma:=\left\|\mathbf{y}_{0}\right\|_{Y}+\|\mathbf{f}\|_{L^{2}\left(0, \infty ; V^{\prime}\right)} \leq \frac{1}{4 C M_{K}^{2}}
$$

there exists a control $u \in L^{2}(0, \infty ; U)$ such that the system

$$
\dot{\mathbf{y}}=A \mathbf{y}+B u-F(\mathbf{y})+\mathbf{f}, \quad \mathbf{y}(0)=\mathbf{y}_{0}
$$

has a unique solution $\mathbf{y} \in W_{\infty}$ satisfying

$$
\|\mathbf{y}\|_{W_{\infty}} \leq 2 M_{K} \gamma \quad \text { and }\|u\|_{L^{2}(0, \infty ; U)} \leq 2\|K\|_{\mathcal{L}(Y)} M_{K} \gamma
$$

Proof By Assumption A2, there exists $K$ such that $A-B K$ generates an exponentially stable, analytic semigroup on $Y$. The result then follows by applying Lemma 5 to the system

$$
\dot{\mathbf{y}}=(A-B K) \mathbf{y}-F(\mathbf{y})+\mathbf{f}, \quad \mathbf{y}(0)=\mathbf{y}_{0} .
$$

and by defining $u=-K \mathbf{y}$.

In the following corollary, we assume without loss of generality that the constant $M_{\lambda}$ given by Consequence $\mathrm{C} 2$ is such that $M_{\lambda} \geq \frac{1}{2 C}$.

Corollary 7 Let $\left(\mathbf{y}_{0}, \mathbf{f}\right) \in Y \times L^{2}\left(0, \infty ; V^{\prime}\right)$ let $u \in L^{2}(0, \infty ; U)$ be such that the system

$$
\dot{\mathbf{y}}=A \mathbf{y}-F(\mathbf{y})+B u+\mathbf{f}, \quad \mathbf{y}(0)=\mathbf{y}_{0}
$$

has a solution $\mathbf{y} \in L^{2}(0, \infty ; Y)$. If

$$
\gamma:=\left\|\mathbf{y}_{0}\right\|_{Y}+\|\mathbf{f}+\lambda \mathbf{y}+B u\|_{L^{2}\left(0, \infty ; V^{\prime}\right)} \leq \frac{1}{4 C M_{\lambda}^{2}},
$$

then $\mathbf{y} \in W_{\infty}$ and it holds that

$$
\|\mathbf{y}\|_{W_{\infty}} \leq 2 M_{\lambda} \gamma
$$

Proof Since $\mathbf{y} \in L^{2}(0, \infty ; Y)$, we can apply Lemma 5 to the equivalent system

$$
\dot{\mathbf{y}}=(A-\lambda I) \mathbf{y}-F(\mathbf{y})+\tilde{\mathbf{f}},
$$

where $\tilde{\mathbf{f}}=\mathbf{f}+\lambda \mathbf{y}+B u$. This shows the assertion. 


\section{Differentiability of the Value Function}

In this section we perform a sensitivity analysis for the stabilization problem. The main purpose is to analyze the dependence of solutions to $(P)$ with respect to the initial condition $\mathbf{y}_{0}$ and to show the differentiability of the associated value function, defined by

$$
\mathcal{V}\left(\mathbf{y}_{0}\right)=\inf _{\substack{\mathbf{y} \in W_{\infty} \\ u \in L^{2}(0, \infty ; U)}} J(\mathbf{y}, u), \quad \text { subject to: } e(\mathbf{y}, u)=\left(0, \mathbf{y}_{0}\right) .
$$

\subsection{Existence of a Solution and Optimality Conditions}

In Lemma 8 we prove the existence of a solution $(\overline{\mathbf{y}}, u)$ to problem $(P)$, assuming that $\left\|\mathbf{y}_{0}\right\|_{Y}$ is sufficiently small. We derive then in Proposition 10 first-order necessary optimality conditions.

Lemma 8 There exists $\delta_{1}>0$ such that for all $\mathbf{y}_{0} \in B_{Y}\left(\delta_{1}\right)$, problem $(P)$ possesses a solution $(\overline{\mathbf{y}}, \bar{u})$. Moreover, there exists a constant $M>0$ independent of $\mathbf{y}_{0}$ such that

$$
\max \left(\|\bar{u}\|_{L^{2}(0, \infty ; U)},\|\overline{\mathbf{y}}\|_{W_{\infty}}\right) \leq M\left\|\mathbf{y}_{0}\right\|_{Y}
$$

Proof Let us set, for the moment, $\delta_{1}=\frac{1}{4 C M_{K}^{2}}$, where $C$ is as in Lemma 4 and $M_{K}$ denotes the constant from Corollary 6 . Applying this corollary (with $\mathbf{f}=0$ ), we obtain that for $\mathbf{y}_{0} \in B_{Y}\left(\delta_{1}\right)$, there exists a control $u \in L^{2}(0, \infty ; U)$ with associated state $\mathbf{y}$ satisfying

$$
\max \left(\|u\|_{L^{2}(0, \infty ; U)},\|\mathbf{y}\|_{W_{\infty}}\right) \leq M\left\|\mathbf{y}_{0}\right\|_{Y}
$$

where $M=2 M_{K} \max \left(1,\|K\|_{\mathcal{L}(Y)}\right)$. We can thus consider a minimizing sequence $\left(\mathbf{y}_{n}, u_{n}\right)_{n \in \mathbb{N}}$ with $J\left(\mathbf{y}_{n}, u_{n}\right) \leq M^{2}\left\|\mathbf{y}_{0}\right\|_{Y}^{2}(1+\alpha)$. We therefore have for all $n \in \mathbb{N}$ that $\left\|\mathbf{y}_{n}\right\|_{L^{2}(0, \infty ; Y)} \leq \sqrt{2} M\left\|\mathbf{y}_{0}\right\|_{Y} \sqrt{1+\alpha}$ and $\left\|u_{n}\right\|_{L^{2}(0, \infty ; U)} \leq \sqrt{2} M\left\|\mathbf{y}_{0}\right\|_{Y} \frac{\sqrt{1+\alpha}}{\sqrt{\alpha}}$.

Possibly after further reduction of $\delta_{1}$, we eventually obtain that

$$
\begin{aligned}
\left\|\mathbf{y}_{0}\right\|_{Y}+\left\|\lambda \mathbf{y}_{n}+B u_{n}\right\|_{L^{2}(0, \infty ; Y)} & \leq\left[1+M \sqrt{2(1+\alpha)}\left(\lambda+\frac{\|B\|_{\mathcal{L}(U, Y)}}{\sqrt{\alpha}}\right)\right] \delta_{1} \\
& \leq \frac{1}{4 C M_{\lambda}^{2}},
\end{aligned}
$$

where $M_{\lambda}$ is as in Corollary 7. It then follows that the sequence $\left(\mathbf{y}_{n}\right)_{n \in \mathbb{N}}$ is bounded in $W_{\infty}$ with $\sup _{n \in \mathbb{N}}\left\|\mathbf{y}_{n}\right\|_{W_{\infty}} \leq 2 M_{\lambda}\left\|\mathbf{y}_{0}\right\|_{Y}$. Extracting if necessary a subsequence, there exists $(\overline{\mathbf{y}}, \bar{u}) \in W_{\infty} \times L^{2}(0, \infty ; U)$ such that $\left(\mathbf{y}_{n}, u_{n}\right) \rightarrow(\overline{\mathbf{y}}, \bar{u}) \in W_{\infty} \times L^{2}(0, \infty ; U)$, and $(\overline{\mathbf{y}}, \bar{u})$ satisfies $(23)$. 
Let us prove that $(\overline{\mathbf{y}}, \bar{u})$ is feasible and optimal. For any $T>0$ let us consider an arbitrary $\mathbf{z} \in H^{1}(0, T ; V)$. For all $n \in \mathbb{N}$, we have

$$
\int_{0}^{T}\left\langle\dot{\mathbf{y}}_{n}(t), \mathbf{z}(t)\right\rangle_{V^{\prime}, V} \mathrm{~d} t=\int_{0}^{T}\left\langle A \mathbf{y}_{n}(t)-F\left(\mathbf{y}_{n}(t)\right)+B u_{n}(t), \mathbf{z}(t)\right\rangle_{V^{\prime}, V} \mathrm{~d} t
$$

Since $\dot{\mathbf{y}}_{n} \rightarrow \dot{\overline{\mathbf{y}}}$ in $L^{2}\left(0, T ; V^{\prime}\right)$, we can pass to the limit in the 1.h.s. of the above equality. Moreover, since $A \mathbf{y}_{n} \rightarrow A \overline{\mathbf{y}} \in L^{2}\left(0, T ; V^{\prime}\right)$,

$$
\int_{0}^{T}\left\langle A \mathbf{y}_{n}(t), \mathbf{z}(t)\right\rangle_{V^{\prime}, V} \mathrm{~d} t \underset{n \rightarrow \infty}{\longrightarrow} \int_{0}^{T}\langle A \overline{\mathbf{y}}(t), \mathbf{z}(t)\rangle_{V^{\prime}, V} \mathrm{~d} t
$$

Analogously, we obtain that

$$
\int_{0}^{T}\left\langle B u_{n}(t), \mathbf{z}(t)\right\rangle_{V^{\prime}, V} \mathrm{~d} t \underset{n \rightarrow \infty}{\longrightarrow} \int_{0}^{T}\langle B \bar{u}(t), \mathbf{z}(t)\rangle_{V^{\prime}, V} \mathrm{~d} t
$$

We also have

$$
\begin{aligned}
& \left|\int_{0}^{T}\left\langle F\left(\mathbf{y}_{n}(t)\right)-F(\overline{\mathbf{y}}(t)), \mathbf{z}(t)\right\rangle_{V^{\prime}, V} \mathrm{~d} t\right| \\
& \quad=\left|\int_{0}^{T}\left\langle N\left(\mathbf{y}_{n}(t), \mathbf{y}_{n}(t)\right)-N(\overline{\mathbf{y}}(t), \overline{\mathbf{y}}(t)), \mathbf{z}(t)\right\rangle_{V^{\prime}, V} \mathrm{~d} t\right| .
\end{aligned}
$$

By Lemma 2, it then follows that

$$
\begin{aligned}
& \left|\int_{0}^{T}\left\langle F\left(\mathbf{y}_{n}(t)\right)-F(\overline{\mathbf{y}}(t)), \mathbf{z}(t)\right\rangle_{V^{\prime}, V} \mathrm{~d} t\right| \\
& \quad \leq M\|\mathbf{z}\|_{L^{\infty}(0, T ; V)}\left\|\mathbf{y}_{n}\right\|_{L^{2}(0, T ; Y)}^{\frac{1}{2}}\left\|\mathbf{y}_{n}-\overline{\mathbf{y}}\right\|_{L^{2}(0, T ; Y)}^{\frac{1}{2}}\left\|\mathbf{y}_{n}\right\|_{L^{2}(0, T ; V)}^{\frac{1}{2}}\left\|\mathbf{y}_{n}-\overline{\mathbf{y}}\right\|_{L^{2}(0, T ; V)}^{\frac{1}{2}} \\
& \quad+M\|\mathbf{z}\|_{L^{\infty}(0, T ; V)}\|\overline{\mathbf{y}}\|_{L^{2}(0, T ; Y)}^{\frac{1}{2}}\left\|\mathbf{y}_{n}-\overline{\mathbf{y}}\right\|_{L^{2}(0, T ; Y)}^{\frac{1}{2}}\|\overline{\mathbf{y}}\|_{L^{2}(0, T ; V)}^{\frac{1}{2}}\left\|\mathbf{y}_{n}-\overline{\mathbf{y}}\right\|_{L^{2}(0, T ; V)}^{\frac{1}{2}}
\end{aligned}
$$

Since $V$ is compactly embedded in $Y$, we obtain that $\left\|\mathbf{y}_{n}-\overline{\mathbf{y}}\right\|_{L^{2}(0, T ; Y)} \underset{n \rightarrow \infty}{\longrightarrow} 0$ with the Aubin-Lions lemma. We can pass to the limit in (24) and obtain

$$
\int_{0}^{T}\langle\dot{\overline{\mathbf{y}}}(t), \mathbf{z}(t)\rangle_{V^{\prime}, V} \mathrm{~d} t=\int_{0}^{T}\langle A \overline{\mathbf{y}}(t)-F(\overline{\mathbf{y}}(t))+B \bar{u}(t), \mathbf{z}(t)\rangle_{V^{\prime}, V} \mathrm{~d} t .
$$

Density of $H^{1}(0, T ; V)$ in $L^{2}(0, T ; V)$ implies that $e(\overline{\mathbf{y}}, \bar{u})=\left(0, \mathbf{y}_{0}\right)$. Finally, by weak lower semi-continuity of norms it follows that $J(\overline{\mathbf{y}}, \bar{u}) \leq \liminf _{n \rightarrow \infty} J\left(\mathbf{y}_{n}, u_{n}\right)$, which proves the optimality of $(\overline{\mathbf{y}}, \bar{u})$. 
Consider now an arbitrary solution $(\tilde{\mathbf{y}}, \tilde{u})$ to $(P)$. It then holds that $J(\tilde{\mathbf{y}}, \tilde{u}) \leq$ $M^{2}\left\|\mathbf{y}_{0}\right\|_{Y}^{2}(1+\alpha)$ from which we obtain that

$$
\|\tilde{\mathbf{y}}\|_{L^{2}(0, \infty ; Y)} \leq \sqrt{2} M\left\|\mathbf{y}_{0}\right\|_{Y} \sqrt{1+\alpha} \text { and }\|\tilde{u}\|_{L^{2}(0, \infty ; U)} \leq \sqrt{2} M\left\|\mathbf{y}_{0}\right\|_{Y} \frac{\sqrt{1+\alpha}}{\sqrt{\alpha}}
$$

The estimate (23) for $\|\tilde{\mathbf{y}}\|_{W_{\infty}}$ can now be shown by applying the same arguments as above.

For the derivation of the optimality system for $(P)$ we need the following technical lemma.

Lemma 9 [15, Lemma 2.5] Let $G \in \mathcal{L}\left(W_{\infty}, L^{2}\left(0, \infty ; V^{\prime}\right)\right)$ be such that $\|G\|<\frac{1}{M_{K}}$, where $\|G\|$ denotes the operator norm of $G$. Then, for all $\mathbf{f} \in L^{2}\left(0, \infty ; V^{\prime}\right)$ and $\mathbf{y}_{0} \in Y$, there exists a unique solution to the following system:

$$
\dot{\mathbf{y}}=(A-B K) \mathbf{y}(t)+(G \mathbf{y})(t)+\mathbf{f}(t), \quad \mathbf{y}(0)=\mathbf{y}_{0} .
$$

Moreover,

$$
\|\mathbf{y}\|_{W_{\infty}} \leq \frac{M_{K}}{1-M_{K}\|G\|}\left(\|\mathbf{f}\|_{L^{2}\left(0, \infty ; V^{\prime}\right)}+\left\|\mathbf{y}_{0}\right\|_{Y}\right) .
$$

First-order optimality conditions for finite-horizon optimal control problems have been addressed several times in the literature, we mention e.g. [1,29-31]. The finitehorizon case, and in particular the decay properties of the state, the costate, and the optimal control, require independent treatment, which we provide next. For an analysis of the linear infinite-horizon problem, we additionally refer to [38].

Proposition 10 There exists $\delta_{2} \in\left(0, \delta_{1}\right]$ such that for all $\mathbf{y}_{0} \in B_{Y}\left(\delta_{2}\right)$, for all solutions $(\overline{\mathbf{y}}, \bar{u})$ of $(P)$, there exists a unique costate $\mathbf{p} \in L^{2}(0, \infty ; V)$ satisfying

$$
\begin{aligned}
-\dot{\mathbf{p}}-A^{*} \mathbf{p}-(\overline{\mathbf{y}} \cdot \nabla) \mathbf{p}+(\nabla \overline{\mathbf{y}})^{T} \mathbf{p} & =\overline{\mathbf{y}} \quad\left(\text { in }\left(W_{\infty}^{0}\right)^{\prime}\right), \\
\alpha \bar{u}+B^{*} \mathbf{p} & =0 .
\end{aligned}
$$

Moreover, there exists a constant $M>0$, independent of $(\overline{\mathbf{y}}, \bar{u})$, such that

$$
\|\mathbf{p}\|_{L^{2}(0, \infty ; V)} \leq M\left\|\mathbf{y}_{0}\right\|_{Y}
$$

Remark 11 Note that (25) is a formal expression for

$$
\begin{aligned}
\left\langle-\dot{\mathbf{p}}-A^{*} \mathbf{p}-(\overline{\mathbf{y}} \cdot \nabla) \mathbf{p}+(\nabla \overline{\mathbf{y}})^{T} \mathbf{p}-\overline{\mathbf{y}}, \mathbf{z}\right\rangle_{\left(W_{\infty}^{0}\right)^{\prime}, W_{\infty}^{0}} \\
=\langle\mathbf{p}, \dot{\mathbf{z}}-A \mathbf{z}+(\mathbf{z} \cdot \nabla) \overline{\mathbf{y}}+(\overline{\mathbf{y}} \cdot \nabla) \mathbf{z}\rangle_{L^{2}(0, \infty ; V), L^{2}\left(0, \infty ; V^{\prime}\right)} \\
\quad-\langle\overline{\mathbf{y}}, \mathbf{z}\rangle_{L^{2}(0, \infty ; Y)}, \quad \forall \mathbf{z} \in W_{\infty}^{0},
\end{aligned}
$$

where $W_{\infty}^{0}:=\left\{\mathbf{z} \in W_{\infty} \mid \mathbf{z}(0)=0\right\}$. 
Proof of Proposition 10 Let us set $\delta_{2}=\delta_{1}$. By Lemma 8, problem $(P)$ has a solution $(\overline{\mathbf{y}}, \bar{u})$. In the first part of the proof, we derive abstract optimality conditions, by proving that the mapping $e$ (used for formulating the constraints) has a surjective derivative. For proving the differentiability of $e$, we only need to consider the nonlinear term. We have $F(\mathbf{y})=N(\mathbf{y}, \mathbf{y})$ and we know that $N$ is a bounded bilinear mapping from $W_{\infty} \times W_{\infty}$ to $L^{2}\left(0, \infty ; V^{\prime}\right)$, by Lemma 2 . Thus $N$ and $F$ are Fréchet differentiable, and so is $e$, with

$$
\begin{aligned}
& D e(\mathbf{y}, u): W_{\infty} \times L^{2}(0, \infty ; U) \rightarrow L^{2}\left(0, \infty ; V^{\prime}\right) \times Y \\
& D e(\mathbf{y}, u)(\mathbf{z}, v)=(\dot{\mathbf{z}}-(A \mathbf{z}-N(\mathbf{y}, \mathbf{z})-N(\mathbf{z}, \mathbf{y})+B v), \mathbf{z}(0)) .
\end{aligned}
$$

Let us show that $\operatorname{De}(\overline{\mathbf{y}}, \bar{u})$ is surjective if $\delta_{2}$ is sufficiently small. Let $(\mathbf{r}, \mathbf{s}) \in$ $L^{2}\left(0, \infty ; V^{\prime}\right) \times Y$ and consider the system

$$
\dot{\mathbf{z}}-(A \mathbf{z}-N(\overline{\mathbf{y}}, \mathbf{z})-N(\mathbf{z}, \overline{\mathbf{y}})+B v)=\mathbf{r}, \quad \mathbf{z}(0)=\mathbf{s} .
$$

Observe that by Corollary 3

$$
\|N(\overline{\mathbf{y}}, \mathbf{z})+N(\mathbf{z}, \overline{\mathbf{y}})\|_{L^{2}\left(0, \infty ; V^{\prime}\right)} \leq M\|\overline{\mathbf{y}}\|_{W_{\infty}}\|\mathbf{z}\|_{W_{\infty}} .
$$

By Lemma 8, it further holds that

$$
\|N(\overline{\mathbf{y}}, \mathbf{z})+N(\mathbf{z}, \overline{\mathbf{y}})\|_{L^{2}\left(0, \infty ; V^{\prime}\right)} \leq M \delta_{2}\|\mathbf{z}\|_{W_{\infty}} .
$$

For sufficiently small $\delta_{2}$, the operator $G \in \mathcal{L}\left(W_{\infty}, L^{2}\left(0, \infty ; V^{\prime}\right)\right)$ defined by

$$
(G \mathbf{z})(t):=D F(\overline{\mathbf{y}}(t))(\mathbf{z}(t))=N(\overline{\mathbf{y}}(t), \mathbf{z}(t))+N(\mathbf{z}(t), \overline{\mathbf{y}}(t))
$$

satisfies $\|G\| \leq \frac{1}{2 M_{K}}<\frac{1}{M_{K}}$. By Lemma 9 there exists a unique solution $\mathbf{z} \in W_{\infty}$ to the system

$$
\dot{\mathbf{z}}-(A-B K) \mathbf{z}+N(\overline{\mathbf{y}}, \mathbf{z})+N(\mathbf{z}, \overline{\mathbf{y}})=\mathbf{r}, \quad \mathbf{z}(0)=\mathbf{s} .
$$

Setting $v=-K \mathbf{z} \in L^{2}(0, \infty ; U)$ proves the surjectivity of $D e(\overline{\mathbf{y}}, \bar{u})$. Note that

$$
\|\mathbf{z}\|_{W_{\infty}} \leq M\left(\|\mathbf{r}\|_{L^{2}\left(0, \infty ; V^{\prime}\right)}+\|\mathbf{s}\|_{L^{2}\left(0, \infty ; V^{\prime}\right)}\right)
$$

for some constant $M$ independent of $(\mathbf{r}, \mathbf{s})$ and $\mathbf{y}_{0}$.

From the surjectivity of $D e(\overline{\mathbf{y}}, \bar{u})$ and Lagrange multiplier theory it follows that there exists a unique pair $(\mathbf{p}, \mu) \in L^{2}(0, \infty ; V) \times Y$ such that for all $(\mathbf{z}, v) \in W_{\infty} \times$ $L^{2}(0, \infty ; U)$,

$$
D J(\overline{\mathbf{y}}, \bar{u})(\mathbf{z}, v)-\langle(\mathbf{p}, \mu), D e(\overline{\mathbf{y}}, \bar{u})(\mathbf{z}, v)\rangle_{L^{2}(0, \infty ; V) \times Y, L^{2}\left(0, \infty ; V^{\prime}\right) \times Y}=0 .
$$


Using (32) we derive in the second part of the proof the costate equation (25) and relation (26). As can be easily verified, $J$ is differentiable with

$$
D J(\overline{\mathbf{y}}, \bar{u})(\mathbf{z}, v)=\langle\overline{\mathbf{y}}, \mathbf{z}\rangle_{L^{2}(0, \infty ; Y)}+\alpha\langle\bar{u}, v\rangle_{L^{2}(0, \infty ; U)}
$$

Moreover, for all $(\mathbf{z}, v) \in W_{\infty} \times L^{2}(0, \infty ; U)$

$$
\begin{aligned}
& \langle(\mathbf{p}, \mu), \operatorname{De}(\overline{\mathbf{y}}, \bar{u})(\mathbf{z}, v)\rangle_{L^{2}(0, \infty ; V) \times Y, L^{2}\left(0, \infty ; V^{\prime}\right) \times Y} \\
& \quad=\langle\mathbf{p}, \dot{\mathbf{z}}\rangle_{L^{2}(0, \infty ; V), L^{2}\left(0, \infty ; V^{\prime}\right)}-\langle\mathbf{p}, A \mathbf{z}-G \mathbf{z}\rangle_{L^{2}(0, \infty ; V), L^{2}\left(0, \infty ; V^{\prime}\right)} \\
& \quad-\langle\mathbf{p}, B v\rangle_{L^{2}(0, \infty ; Y)}+\langle\mu, \mathbf{z}(0)\rangle_{Y}
\end{aligned}
$$

Taking $\mathbf{z}=0$ and letting $v$ vary in $L^{2}(0, \infty ; U)$, we deduce from (32), (33) and (34) that

$$
\alpha \bar{u}+B^{*} \mathbf{p}=0 \text { in } L^{2}(0, \infty ; U),
$$

which proves (26). Taking now $v=0$, we obtain that

$$
\begin{aligned}
\langle\mathbf{p}, \dot{\mathbf{z}}\rangle_{L^{2}(0, \infty ; V), L^{2}\left(0, \infty ; V^{\prime}\right)}= & \langle\mathbf{p}, A \mathbf{z}-G \mathbf{z}\rangle_{L^{2}(0, \infty ; V), L^{2}\left(0, \infty ; V^{\prime}\right)} \\
& +\langle\overline{\mathbf{y}}, \mathbf{z}\rangle_{L^{2}(0, \infty ; Y)}, \quad \forall \mathbf{z} \in W_{\infty}^{0}
\end{aligned}
$$

It remains to bound $\mathbf{p}$ in $L^{2}(0, \infty ; V)$. Let $\mathbf{r} \in L^{2}\left(0, \infty ; V^{\prime}\right)$ and let $(\mathbf{z}, v)$ satisfy $\operatorname{De}(\overline{\mathbf{y}}, \bar{u})(\mathbf{z}, v)=(\mathbf{r}, 0)$ and the bound (31) (with $\mathbf{s}=0)$. Using the optimality condition (32), the expression (33) of $D J(\overline{\mathbf{y}}, \bar{u})$, estimate (31), and estimate (23) on $(\overline{\mathbf{y}}, \bar{u})$, we obtain the following inequalities:

$$
\begin{aligned}
\langle\mathbf{p}, & \mathbf{r}\rangle_{L^{2}(0, \infty ; V), L^{2}\left(0, \infty ; V^{\prime}\right)} \\
& =\langle(\mathbf{p}, \mu),(\mathbf{r}, 0)\rangle_{L^{2}(0, \infty ; V) \times Y, L^{2}\left(0, \infty ; V^{\prime}\right) \times Y} \\
& =\left\langle\operatorname{De}(\overline{\mathbf{y}}, \bar{u})^{\prime}(\mathbf{p}, \mu),(\mathbf{z}, v)\right\rangle_{W_{\infty}^{\prime} \times L^{2}(0, \infty ; U), W_{\infty} \times L^{2}(0, \infty ; U)} \\
& =\operatorname{DJ}(\overline{\mathbf{y}}, \bar{u})(\mathbf{z}, v) \\
& \leq M\left(\|\overline{\mathbf{y}}\|_{L^{2}(0, \infty ; Y)}+\|\bar{u}\|_{L^{2}(0, \infty ; U)}\right)\left(\|\mathbf{z}\|_{L^{2}(0, \infty ; Y)}+\|v\|_{L^{2}(0, \infty ; U)}\right) \\
& \leq M\left\|\mathbf{y}_{0}\right\|_{Y}\|\mathbf{r}\|_{L^{2}\left(0, \infty ; V^{\prime}\right)} .
\end{aligned}
$$

Since $\mathbf{r}$ was arbitrary and since $M$ does not depend on $\mathbf{r}$, we obtain that $\|\mathbf{p}\|_{L^{2}(0, \infty ; V)} \leq$ $M\left\|\mathbf{y}_{0}\right\|_{Y}$. 


\subsection{Sensitivity Analysis}

We define a mapping $\Phi$ via

$$
\begin{aligned}
\Phi: W_{\infty} & \times L^{2}(0, \infty ; U) \times L^{2}(0, \infty ; V) \rightarrow Y \times L^{2}\left(0, \infty ; V^{\prime}\right) \times\left(W_{\infty}^{0}\right)^{\prime} \\
& \times L^{2}(0, \infty ; U)=: X, \\
& \\
& (\mathbf{y}, u, \mathbf{p})=\left(\begin{array}{c}
\mathbf{y}(0) \\
\dot{\mathbf{y}}-A \mathbf{y}+F(\mathbf{y})-B u \\
-\dot{\mathbf{p}}-A^{*} \mathbf{p}-(\mathbf{y} \cdot \nabla) \mathbf{p}+(\nabla \mathbf{y})^{T} \mathbf{p}-\mathbf{y} \\
\alpha u+B^{*} \mathbf{p}
\end{array}\right)
\end{aligned}
$$

where the third line again has to be understood formally, see Remark 11 . We endow the space $X$ with the $l_{\infty}$-product norm. The well-posedness of $\Phi$ follows from the considerations on $e(\mathbf{y}, u)$ and the costate Eq. (25) that have been given in the proof of Proposition 10.

Lemma 12 There exist $\delta_{3}>0, \delta_{3}^{\prime}>0$, and three $C^{\infty}$-mappings

$$
\mathbf{y}_{0} \in B_{Y}\left(\delta_{3}\right) \mapsto\left(\mathcal{Y}\left(\mathbf{y}_{0}\right), \mathcal{U}\left(\mathbf{y}_{0}\right), \mathcal{P}\left(\mathbf{y}_{0}\right)\right) \in W_{\infty} \times L^{2}(0, \infty ; U) \times L^{2}(0, \infty ; V)
$$

such that for all $\mathbf{y}_{0} \in B_{Y}\left(\delta_{3}\right)$, the triplet $\left(\mathcal{Y}\left(\mathbf{y}_{0}\right), \mathcal{U}\left(\mathbf{y}_{0}\right), \mathcal{P}\left(\mathbf{y}_{0}\right)\right)$ is the unique solution to

$$
\Phi(\mathbf{y}, u, \mathbf{p})=\left(\mathbf{y}_{0}, 0,0,0\right), \quad \max \left(\|\mathbf{y}\|_{W_{\infty}},\|u\|_{L^{2}(0, \infty ; U)},\|\mathbf{p}\|_{L^{2}(0, \infty ; V)}\right) \leq \delta_{3}^{\prime}
$$

in $W_{\infty} \times L^{2}(0, \infty ; U) \times L^{2}(0, \infty ; V)$. Moreover, there exists a constant $M>0$ such that for all $\mathbf{y}_{0} \in B_{Y}\left(\delta_{3}\right)$,

$$
\max \left(\left\|\mathcal{Y}\left(\mathbf{y}_{0}\right)\right\|_{W_{\infty}},\left\|\mathcal{U}\left(\mathbf{y}_{0}\right)\right\|_{L^{2}(0, \infty ; U)},\left\|\mathcal{P}\left(\mathbf{y}_{0}\right)\right\|_{L^{2}(0, \infty ; V)}\right) \leq M\left\|\mathbf{y}_{0}\right\|_{Y} .
$$

Proof The result is a consequence of the inverse function theorem. Since $\Phi$ contains only linear terms and three bilinear terms, it is infinitely differentiable. We also have $\Phi(0,0,0)=(0,0,0,0)$. It remains to prove that $D \Phi(0,0,0)$ is an isomorphism. Let $\left(\mathbf{w}_{1}, \mathbf{w}_{2}, \mathbf{w}_{3}, w_{4}\right) \in X$ and let $(\mathbf{y}, u, \mathbf{p}) \in W_{\infty} \times L^{2}(0, \infty ; U) \times L^{2}(0, \infty ; V)$. We have the following equivalence

$$
D \Phi(0,0,0)(\mathbf{y}, u, \mathbf{p})=\left(\mathbf{w}_{1}, \mathbf{w}_{2}, \mathbf{w}_{3}, w_{4}\right) \Longleftrightarrow\left\{\begin{array}{r}
\mathbf{y}(0)=\mathbf{w}_{1} \\
\dot{\mathbf{y}}-A \mathbf{y}-B u=\mathbf{w}_{2} \\
-\dot{\mathbf{p}}-A^{*} \mathbf{p}-\mathbf{y}=\mathbf{w}_{3} \\
\alpha u+B^{*} \mathbf{p}=w_{4}
\end{array}\right.
$$

It can be proved with the same techniques as for [15, Proposition 3.1, Lemma 4.4] that the linear system on the left-hand side has a unique solution $(\mathbf{y}, u, \mathbf{p})$, moreover,

$$
\|(\mathbf{y}, u, \mathbf{p})\|_{W_{\infty} \times L^{2}(0, \infty ; U) \times L^{2}(0, \infty ; V)} \leq M\left\|\left(\mathbf{w}_{1}, \mathbf{w}_{2}, \mathbf{w}_{3}, w_{4}\right)\right\|_{X} .
$$


This proves that $D \Phi(0,0,0)$ is an isomorphism. The inverse function theorem ensures the existence of $\delta_{3}>0, \delta_{3}^{\prime}>0$, and $C^{\infty}$-mappings $\mathcal{Y}, \mathcal{U}$, and $\mathcal{P}$ with the properties announced in (37).

It remains to prove (38). Reducing if necessary $\delta_{3}$, we can assume that the norms of the derivatives of the three mappings are bounded on $B_{Y}\left(\delta_{3}\right)$ by some constant $M>0$. The three mappings are therefore Lipschitz continuous with modulus $M$. Estimate (38) follows, since $(\mathcal{Y}(0), \mathcal{U}(0),(\mathcal{P}(0))=(0,0,0)$.

Proposition 13 There exists $\delta_{4} \in\left(0, \min \left(\delta_{2}, \delta_{3}\right)\right]$ such that for all $\mathbf{y}_{0} \in B_{Y}\left(\delta_{4}\right)$, the pair $\left(\mathcal{Y}\left(y_{0}\right), \mathcal{U}\left(y_{0}\right)\right)$ is the unique solution to $(P)$ with initial condition $\mathbf{y}_{0}$. Moreover, $\mathcal{P}\left(\mathbf{y}_{0}\right)$ is the unique associated costate.

Proof Let us set $\delta_{4}=\min \left(\delta_{2}, \delta_{3}\right)$ for the moment. Let $\mathbf{y}_{0} \in B_{Y}\left(\delta_{4}\right)$. By Lemma 8 and Proposition 10 , there exist a solution $(\overline{\mathbf{y}}, \bar{u})$ to $(P)$ with associated costate $\overline{\mathbf{p}}$ which necessarily satisfies

$$
\max \left(\|\overline{\mathbf{y}}\|_{W_{\infty}},\|\bar{u}\|_{L^{2}(0, \infty ; U)},\|\overline{\mathbf{p}}\|_{L^{2}(0, \infty ; V)}\right) \leq M\left\|\mathbf{y}_{0}\right\|_{Y}
$$

By further reduction of $\delta_{4}$, we obtain that

$$
\max \left(\|\overline{\mathbf{y}}\|_{W_{\infty}},\|\bar{u}\|_{L^{2}(0, \infty ; U)},\|\overline{\mathbf{p}}\|_{L^{2}(0, \infty ; V)}\right) \leq \delta_{3}^{\prime} .
$$

Since $\Phi(\overline{\mathbf{y}}, \bar{u}, \overline{\mathbf{p}})=\left(\mathbf{y}_{0}, 0,0,0\right)$, Lemma 12 implies that $(\overline{\mathbf{y}}, \bar{u}, \overline{\mathbf{p}})=\left(\mathcal{Y}\left(\mathbf{y}_{0}\right)\right.$, $\left.\mathcal{U}\left(\mathbf{y}_{0}\right), \mathcal{P}\left(\mathbf{y}_{0}\right)\right)$. The proposition is proved.

Corollary 14 The value function $\mathcal{V}$ is infinitely differentiable on $B_{Y}\left(\delta_{4}\right)$.

Proof The cost function $J$ is clearly infinitely differentiable. Since $\mathcal{V}\left(\mathbf{y}_{0}\right)=$ $J\left(\mathcal{Y}\left(\mathbf{y}_{0}\right), \mathcal{U}\left(\mathbf{y}_{0}\right)\right), \mathcal{V}$ is then the composition of infinitely differentiable mappings, which shows the assertion.

\subsection{Additional Regularity for $p$}

We next assert that for small initial data $\mathbf{y}_{0}$ the adjoint state is more regular than $\mathbf{p} \in L^{2}(0, \infty ; V)$. For this, we need more smoothness of the boundary $\Gamma$.

Assumption A3 Let $\Omega \subset \mathbb{R}^{2}$ denote a bounded domain with smooth boundary $\Gamma$.

Proposition 15 There exists $\tilde{\delta}_{4} \in\left(0, \delta_{4}\right]$ such that for all $\mathbf{y}_{0} \in B_{Y}\left(\tilde{\delta}_{4}\right)$, for all solutions $(\overline{\mathbf{y}}, \bar{u})$ of $(P)$, there exists a unique costate $\mathbf{p} \in W_{\infty}$ satisfying

$$
-\dot{\mathbf{p}}-A^{*} \mathbf{p}-(\overline{\mathbf{y}} \cdot \nabla) \mathbf{p}+(\nabla \overline{\mathbf{y}})^{T} \mathbf{p}=\overline{\mathbf{y}} \quad\left(\text { in } L^{2}\left(0, \infty ; V^{\prime}\right)\right) .
$$

Moreover, there exists a constant $M>0$, independent of $(\overline{\mathbf{y}}, \bar{u})$, such that

$$
\|\mathbf{p}\|_{W_{\infty}} \leq M\left\|\mathbf{y}_{0}\right\|_{Y}
$$

The proof is given in the Appendix. 


\section{Derivatives of the Value Function}

By standard arguments, we can derive a Hamilton-Jacobi-Bellman equation which provides an optimal feedback control based on the derivative of the value function.

All along the section, the first-order derivative $D \mathcal{V}\left(\mathbf{y}_{0}\right)$ is either seen as a linear form on $Y$ or is identified with its Riesz representative in $Y$. The identification is done for example in the term $\left\|B^{*} D \mathcal{V}\left(\mathbf{y}_{0}\right)\right\|_{U}^{2}$ appearing in the HJB equation below.

Proposition 16 There exists $\delta_{5} \in\left(0, \tilde{\delta}_{4}\right]$ such that for all $\mathbf{y}_{0} \in B_{Y}\left(\delta_{5}\right) \cap \mathcal{D}(A)$, the following Hamilton-Jacobi-Bellman equation holds:

$$
D \mathcal{V}\left(\mathbf{y}_{0}\right)\left(A \mathbf{y}_{0}-F\left(\mathbf{y}_{0}\right)\right)+\frac{1}{2}\left\|\mathbf{y}_{0}\right\|_{Y}^{2}-\frac{1}{2 \alpha}\left\|B^{*} D \mathcal{V}\left(\mathbf{y}_{0}\right)\right\|_{U}^{2}=0 .
$$

Moreover,

$$
\bar{u}(t)=-\frac{1}{\alpha} B^{*} D \mathcal{V}(\overline{\mathbf{y}}(t)), \text { for all } t \geq 0,
$$

where $(\overline{\mathbf{y}}, \bar{u})=\left(\mathcal{Y}\left(\mathbf{y}_{0}\right), \mathcal{U}\left(\mathbf{y}_{0}\right)\right)$.

Remark 17 Note that by, e.g., [6, Proposition 1.7], we have that $F: \mathcal{D}(A) \times \mathcal{D}(A) \rightarrow Y$ and, as a consequence, the term $D \mathcal{V}\left(\mathbf{y}_{0}\right) F\left(\mathbf{y}_{0}\right)$ is well-defined.

Proof Let us set $\delta_{5}=\delta_{4}$. Let $\mathbf{y}_{0} \in B_{Y}\left(\delta_{5}\right) \cap \mathcal{D}(A)$. Let us consider the Hamiltonian of the system, defined by

$$
\begin{aligned}
H\left(\mathbf{y}_{0}, u, \mathbf{p}\right)= & \frac{1}{2}\|\mathbf{y}\|_{Y}^{2}+\frac{\alpha}{2}\|u\|_{U}^{2}+\langle\mathbf{p}, A \mathbf{y}-F(\mathbf{y}) \\
& +B u\rangle_{Y}, \quad \forall(\mathbf{y}, u, \mathbf{p}) \in \mathcal{D}(A) \times U \times Y .
\end{aligned}
$$

Using the arguments provided in the proof of [17, Proposition 9], one can prove that

$$
\min _{u \in U} H\left(\mathbf{y}_{0}, u, D \mathcal{V}\left(\mathbf{y}_{0}\right)\right)=0
$$

from which (42) derives. One can also prove that

$$
\bar{u}(0)=\arg \min _{u \in U} H\left(\mathbf{y}_{0}, u, D \mathcal{V}\left(\mathbf{y}_{0}\right)\right),
$$

which proves (43) for $t=0$. Let us emphasize that the assumptions which are required in [17, Proposition 9] are satisfied. In particular, the optimality condition $\bar{u}(t)=$ $-\frac{1}{\alpha} B^{*} \overline{\mathbf{p}}(t)$ which holds in $L^{2}(0, \infty ; U)$ implies that $\bar{u}$ is almost everywhere equal to a continuous function. We can thus assume that $\bar{u}$ is continuous. For proving (43) for all $t \geq 0$, one has first to reduce $\delta_{5}$ so that $\|\overline{\mathbf{y}}(t)\|_{Y} \leq \delta_{4}$, for all $t \geq 0$. For a given $t \geq 0$, we have by dynamic programming that $(\overline{\mathbf{y}}(t+\cdot), \bar{u}(t+\cdot))$ is the solution to $(P)$ with initial condition $\overline{\mathbf{y}}(t)$ and thus (43) holds true at $t$. 
For deriving a Taylor series expansion of $\mathcal{V}$, let us follow the approach from [3] and differentiate (42) in some direction $\mathbf{z}_{1} \in \mathcal{D}(A)$. To alleviate the calculations, we denote the variable $\mathbf{y}_{0}$ in (42) by $\mathbf{y}$. We then obtain

$$
\begin{gathered}
D^{2} \mathcal{V}(\mathbf{y})\left(A \mathbf{y}-F(\mathbf{y}), \mathbf{z}_{1}\right)+D \mathcal{V}(\mathbf{y})\left(A \mathbf{z}_{1}-A_{0}\left(\mathbf{y}, \mathbf{z}_{1}\right)\right)+\left\langle\mathbf{y}, \mathbf{z}_{1}\right\rangle_{Y} \\
-\frac{1}{\alpha}\left\langle B^{*} D^{2} \mathcal{V}(\mathbf{y})\left(\cdot, \mathbf{z}_{1}\right), B^{*} D \mathcal{V}(\mathbf{y})\right\rangle_{U}=0 .
\end{gathered}
$$

A second differentiation in the directions $\left(\mathbf{z}_{1}, \mathbf{z}_{2}\right) \in \mathcal{D}(A)^{2}$ yields the equation

$$
\begin{aligned}
& D^{3} \mathcal{V}(\mathbf{y})\left(A \mathbf{y}-F(\mathbf{y}), \mathbf{z}_{1}, \mathbf{z}_{2}\right)+D^{2} \mathcal{V}(\mathbf{y})\left(A \mathbf{z}_{2}-A_{0}\left(\mathbf{y}, \mathbf{z}_{2}\right), \mathbf{z}_{1}\right) \\
& \quad+D^{2} \mathcal{V}(\mathbf{y})\left(A \mathbf{z}_{1}-A_{0}\left(\mathbf{y}, \mathbf{z}_{1}\right), \mathbf{z}_{2}\right) \\
& \quad-D \mathcal{V}(\mathbf{y})\left(A_{0}\left(\mathbf{z}_{2}, \mathbf{z}_{1}\right)\right)+\left\langle\mathbf{z}_{2}, \mathbf{z}_{1}\right\rangle_{Y}-\frac{1}{\alpha}\left\langle B^{*} D^{3} \mathcal{V}(\mathbf{y})\left(\cdot, \mathbf{z}_{1}, \mathbf{z}_{2}\right), B^{*} D \mathcal{V}(\mathbf{y})\right\rangle_{U} \\
& \quad-\frac{1}{\alpha}\left\langle B^{*} D^{2} \mathcal{V}(\mathbf{y})\left(\cdot, \mathbf{z}_{1}\right), B^{*} D^{2} \mathcal{V}(\mathbf{y})\left(\cdot, \mathbf{z}_{2}\right)\right\rangle_{U}=0 .
\end{aligned}
$$

Since $\mathcal{V}(0)=0$ and $\mathcal{V}(\mathbf{y}) \geq 0$ for all $\mathbf{y} \in Y$, it follows that $D \mathcal{V}(0)=0$. We can thus evaluate the last equation for $\mathbf{y}=0$ to obtain

$$
\begin{aligned}
& D^{2} \mathcal{V}(0)\left(A \mathbf{z}_{2}, \mathbf{z}_{1}\right)+D^{2} \mathcal{V}(0)\left(A \mathbf{z}_{1}, \mathbf{z}_{2}\right)+\left\langle\mathbf{z}_{2}, \mathbf{z}_{1}\right\rangle_{Y} \\
& -\frac{1}{\alpha}\left\langle B^{*} D^{2} \mathcal{V}(0)\left(\cdot, \mathbf{z}_{1}\right), B^{*} D^{2} \mathcal{V}(0)\left(\cdot, \mathbf{z}_{2}\right)\right\rangle_{U}=0 .
\end{aligned}
$$

We recall that $D^{2} \mathcal{V}(0) \in \mathcal{M}(Y \times Y, \mathbb{R})$ is a bounded and symmetric bilinear form on $Y$ and thus can be represented (see, e.g., [32, Chapter 5, Section 2]) by an operator $\Pi \in \mathcal{L}(Y)$ such that

$$
D^{2} \mathcal{V}(0)(\mathbf{y}, \mathbf{z})=\langle\Pi \mathbf{y}, \mathbf{z}\rangle_{Y}, \text { for all } \mathbf{y}, \mathbf{z} \in Y
$$

As a consequence, we can formulate (44) as

$$
\left\langle\mathbf{z}_{2}, A^{*} \Pi \mathbf{z}_{1}\right\rangle_{Y}+\left\langle\Pi A \mathbf{z}_{1}, \mathbf{z}_{2}\right\rangle_{Y}+\left\langle\mathbf{z}_{2}, \mathbf{z}_{1}\right\rangle_{Y}-\frac{1}{\alpha}\left\langle B^{*} \Pi \mathbf{z}_{1}, B^{*} \Pi \mathbf{z}_{2}\right\rangle_{U}=0 .
$$

Equation (45) is the well-known algebraic operator Riccati equation which has been studied in detail in, e.g., [20,33]. From the stabilizability Assumption A2, and the fact that the pair $(A, \mathrm{id})$ is exponentially detectable as a consequence of $(18)$, we conclude that (45) has a unique stabilizing solution $\Pi \in \mathcal{L}(Y)$. In the discussion below, we denote by

$$
A_{\pi}:=A-\frac{1}{\alpha} B B^{*} \Pi
$$


the closed-loop operator associated with the linearized stabilization problem. In particular, let us mention that $A_{\pi}$ generates an analytic exponentially stable semigroup $e^{A_{\pi} t}$ on $Y$. Hence, for trajectories of the form $\tilde{\mathbf{y}}=e^{A \cdot \mathbf{y}}, \mathbf{y} \in Y$ it follows that $\tilde{\mathbf{y}} \in W_{\infty}$.

For higher order derivatives of $\mathcal{V}$, we follow the exposition from [17]. For this purpose, let us briefly recall the symmetrization technique introduced there. Let $i$ and $j \in \mathbb{N}$, consider

$$
S_{i, j}=\left\{\sigma \in S_{i+j} \mid \sigma(1)<\cdots<\sigma(i) \text { and } \sigma(i+1)<\cdots<\sigma(i+j)\right\},
$$

where $S_{i+j}$ is the set of permutations of $\{1, \ldots, i+j\}$. A permutation $\sigma \in S_{i, j}$ is uniquely defined by the subset $\{\sigma(1), \ldots, \sigma(i)\}$, therefore, the cardinality of $S_{i, j}$ is equal to the number of subsets of cardinality $i$ of $\{1, \ldots, i+j\}$, that is to say $\left|S_{i, j}\right|=\left(\begin{array}{c}i+j \\ i\end{array}\right)$. For a multilinear mapping $\mathcal{T}$ of order $i+j$, we set

$$
\operatorname{Sym}_{i, j}(\mathcal{T})\left(\mathbf{z}_{1}, \ldots, \mathbf{z}_{i+j}\right)=\left(\begin{array}{c}
i+j \\
i
\end{array}\right)^{-1}\left[\sum_{\sigma \in S_{i, j}} \mathcal{T}\left(\mathbf{z}_{\sigma(1)}, \ldots, \mathbf{z}_{\sigma(i+j)}\right)\right]
$$

The following proposition is a generalization of the Leibniz formula for the differentiation of the product of two functions.

Proposition 18 Let $Z$ be a Hilbert space. Let $f: Y \rightarrow Z$ and $g: Y \rightarrow Z$ be two $k$-times continuously differentiable functions. Then, for all $k \geq 1$, for all $\mathbf{y} \in Y$ and $\left(\mathbf{z}_{1}, \ldots, \mathbf{z}_{k}\right) \in Y^{k}$

$$
\begin{aligned}
D^{k} & {\left[\langle f(\mathbf{y}), g(\mathbf{y})\rangle_{Z}\right]\left(\mathbf{z}_{1}, \ldots, \mathbf{z}_{k}\right) } \\
& =\sum_{i=0}^{k}\left(\begin{array}{l}
k \\
i
\end{array}\right) \operatorname{Sym}_{i, k-i}\left(D^{i} f(\mathbf{y}) \otimes D^{k-i} g(\mathbf{y})\right)\left(\mathbf{z}_{1}, \ldots, \mathbf{z}_{k}\right) .
\end{aligned}
$$

Proof The proof is analogous to the one given in [17, Lemma 10] for $Z=\mathbb{R}$.

Theorem 19 Let $k \geq 3$. For all $\mathbf{z}_{1}, \ldots, \mathbf{z}_{k} \in \mathcal{D}(A)$,

$$
\sum_{i=1}^{k} \mathcal{D}^{k} \mathcal{V}(0)\left(\mathbf{z}_{1}, \ldots, \mathbf{z}_{i-1}, A_{\pi} \mathbf{z}_{i}, \mathbf{z}_{i+1}, \ldots, \mathbf{z}_{k}\right)=\mathcal{R}_{k}\left(\mathbf{z}_{1}, \ldots, \mathbf{z}_{k}\right)
$$

where the multilinear form $\mathcal{R}_{k}: \mathcal{D}(A)^{k} \rightarrow \mathbb{R}$ is given by

$$
\begin{aligned}
\mathcal{R}_{k}\left(\mathbf{z}_{1}, \ldots, \mathbf{z}_{k}\right)= & \frac{1}{2 \alpha} \sum_{i=2}^{k-2}\left(\begin{array}{c}
k \\
i
\end{array}\right) \operatorname{Sym}_{i, k-i}\left(\mathcal{C}_{i} \otimes \mathcal{C}_{k-i}\right)\left(\mathbf{z}_{1}, \ldots, \mathbf{z}_{k}\right) \\
& +\frac{k(k-1)}{2} \underset{k-2,2}{\operatorname{Sym}}\left(D^{k-1} \mathcal{V}(0) \otimes D^{2} F(0)\right)\left(\mathbf{z}_{1}, \ldots, \mathbf{z}_{k}\right)
\end{aligned}
$$

with $\mathcal{C}_{i}\left(\mathbf{z}_{1}, \ldots, \mathbf{z}_{i}\right)=B^{*} D^{i+1} \mathcal{V}(0)\left(\cdot, \mathbf{z}_{1}, \ldots, \mathbf{z}_{i}\right)$ and $D^{2} F(0)\left(\mathbf{z}_{1}, \mathbf{z}_{2}\right)=A_{0}\left(\mathbf{z}_{1}, \mathbf{z}_{2}\right)$ 
Proof The proof relies on successive differentiations of (42). For a bilinear control problem, a similar result has been obtained in [17, Theorem 12]. In particular, it was shown that

$$
\left(D^{k}[\mathcal{V}(\mathbf{y})(A \mathbf{y})]_{\mathbf{y}=0}\right)\left(\mathbf{z}_{1}, \ldots, \mathbf{z}_{k}\right)=\sum_{i=1}^{k} D^{k} \mathcal{V}(0)\left(\mathbf{z}_{1}, \ldots, \mathbf{z}_{i-1}, A \mathbf{z}_{i}, \mathbf{z}_{i+1}, \ldots, \mathbf{z}_{k}\right)
$$

Obviously, for $k \geq 3$, we have $D^{k}\left(\frac{1}{2}\|\mathbf{y}\|_{Y}^{2}\right)=0$. Let us discuss the structure of the derivatives of the remaining terms appearing in (42). Applying Proposition 18 to the term $\left\|B^{*} D \mathcal{V}(\mathbf{y})\right\|_{U}^{2}$, we obtain

$$
D^{k}\left\|B^{*} D \mathcal{V}(\mathbf{y})\right\|_{U}^{2}=\sum_{i=0}^{k}\left(\begin{array}{l}
k \\
i
\end{array}\right) \operatorname{Sym}_{i, k-i}\left(D^{i}\left(B^{*} D \mathcal{V}(\mathbf{y})\right) \otimes D^{k-i}\left(B^{*} D \mathcal{V}(\mathbf{y})\right)\right)
$$

Since $\mathcal{V}$ has a minimum at the origin, we have $D \mathcal{V}(0)=0$ and the terms for $i=0$ and $i=k$ vanish when evaluated in $\mathbf{y}=0$. By definition of the Sym-operator, for $i=1$ we obtain

$$
\begin{aligned}
\left(\begin{array}{c}
k \\
1
\end{array}\right) & \left.\operatorname{Sym}_{1, k-1}\left(D\left(B^{*} D \mathcal{V}(\mathbf{y})\right) \otimes D^{k-1}\left(B^{*} D \mathcal{V}(\mathbf{y})\right)\right)\left(\mathbf{z}_{1}, \ldots, \mathbf{z}_{k}\right)\right|_{\mathbf{y}=0} \\
= & \sum_{\sigma \in S_{1, k-1}}\left\langle B^{*} D^{2} \mathcal{V}(0)\left(\cdot, \mathbf{z}_{\sigma(1)}\right), B^{*} D^{k} \mathcal{V}(0)\left(\cdot, \mathbf{z}_{\sigma(2)}, \ldots, \mathbf{z}_{\sigma(k)}\right)\right\rangle_{U} \\
= & \sum_{\sigma \in S_{1, k-1}}\left\langle B B^{*} D^{2} \mathcal{V}(0)\left(\cdot, \mathbf{z}_{\sigma(1)}\right), D^{k} \mathcal{V}(0)\left(\cdot, \mathbf{z}_{\sigma(2)}, \ldots, \mathbf{z}_{\sigma(k)}\right)\right\rangle_{Y} \\
= & \sum_{\sigma \in S_{1, k-1}} D^{k} \mathcal{V}(0)\left(B B^{*} D^{2} \mathcal{V}(0)\left(\cdot, \mathbf{z}_{\sigma(1)}\right), \mathbf{z}_{\sigma(2)}, \ldots, \mathbf{z}_{\sigma(k)}\right)
\end{aligned}
$$

As explained previously, we can represent $D^{2} \mathcal{V}(0)$ in terms of the solution $\Pi$ of the algebraic operator Riccati equation. This shows

$$
\begin{aligned}
\left(\begin{array}{c}
k \\
1
\end{array}\right) & \left.\operatorname{Sym}_{1, k-1}\left(D\left(B^{*} D \mathcal{V}(\mathbf{y})\right) \otimes D^{k-1}\left(B^{*} D \mathcal{V}(\mathbf{y})\right)\right)\left(\mathbf{z}_{1}, \ldots, \mathbf{z}_{k}\right)\right|_{\mathbf{y}=0} \\
= & \sum_{\sigma \in S_{1, k-1}} D^{k} \mathcal{V}(0)\left(B B^{*} \Pi \mathbf{z}_{\sigma(1)}, \mathbf{z}_{\sigma(2)}, \ldots, \mathbf{z}_{\sigma(k)}\right) \\
= & \sum_{i=1}^{k} D^{k} \mathcal{V}(0)\left(\mathbf{z}_{1}, \ldots, \mathbf{z}_{i-1}, B B^{*} \mathbf{z}_{i}, \mathbf{z}_{i+1}, \ldots, \mathbf{z}_{k}\right)
\end{aligned}
$$

A similar relation can be derived for $i=k-1$. Finally we consider the term $D^{k}(D \mathcal{V}(\mathbf{y}) F(\mathbf{y}))$. By Proposition 18, we get 


$$
\begin{aligned}
D^{k} & (D \mathcal{V}(\mathbf{y}) F(\mathbf{y}))\left(\mathbf{z}_{1}, \ldots, \mathbf{z}_{k}\right) \\
& =D^{k}\langle D \mathcal{V}(\mathbf{y}), F(\mathbf{y})\rangle_{Y}\left(\mathbf{z}_{1}, \ldots, \mathbf{z}_{k}\right) \\
& =\sum_{i=0}^{k}\left(\begin{array}{c}
k \\
i
\end{array}\right) \underset{i, k-i}{\operatorname{Sym}}\left(D^{i+1} \mathcal{V}(\mathbf{y}) \otimes D^{k-i} F(\mathbf{y})\right)\left(\mathbf{z}_{1}, \ldots, \mathbf{z}_{k}\right) .
\end{aligned}
$$

Since $D^{3+\ell} F(\mathbf{y})=0$ for all $\ell \geq 0$, the previous equation simplifies as follows

$$
\begin{aligned}
D^{k} & (D \mathcal{V}(\mathbf{y}) F(\mathbf{y}))\left(\mathbf{z}_{1}, \ldots, \mathbf{z}_{k}\right) \\
& =\sum_{i=k-2}^{k}\left(\begin{array}{l}
k \\
i
\end{array}\right) \operatorname{Sym}_{i, k-i}\left(D^{i+1} \mathcal{V}(\mathbf{y}) \otimes D^{k-i} F(\mathbf{y})\right)\left(\mathbf{z}_{1}, \ldots, \mathbf{z}_{k}\right) .
\end{aligned}
$$

Evaluating the last expression in $\mathbf{y}=0$ yields

$$
\begin{aligned}
& \left(D^{k}[D \mathcal{V}(\mathbf{y}) F(\mathbf{y})]_{\mathbf{y}=0}\right)\left(\mathbf{z}_{1}, \ldots, \mathbf{z}_{k}\right) \\
& =\frac{k(k-1)}{2} \operatorname{Sym}_{k-2,2}\left(D^{k-1} \mathcal{V}(0) \otimes D^{2} F(0)\right)\left(\mathbf{z}_{1}, \ldots, \mathbf{z}_{k}\right),
\end{aligned}
$$

since $F(0)$ and $D F(0)$ are both null. Combining (48), (49) and (50) proves the assertion.

\section{Polynomial Feedback Laws}

\subsection{Estimates for the Velocity}

In this section we analyze the polynomial feedback law $u_{d}$ derived from the Taylor series approximation of the value function

$$
\mathcal{V}_{d}(\mathbf{y}):=\sum_{k=2}^{d} \frac{1}{k !} D^{k} \mathcal{V}(0)(\mathbf{y}, \ldots, \mathbf{y})
$$

for a given $d \geq 2$. The feedback $u_{d}: Y \rightarrow U$ is obtained by approximating $\mathcal{V}$ with $\mathcal{V}_{d}$ in formula (43), that is

$$
u_{d}(\mathbf{y})=-\frac{1}{\alpha} B^{*} D \mathcal{V}_{d}(\mathbf{y})=-\frac{1}{\alpha} \sum_{k=2}^{d} \frac{1}{(k-1) !} B^{*} D^{k} \mathcal{V}(0)(\cdot, \mathbf{y}, \ldots, \mathbf{y})
$$

The associated closed-loop system is given by

$$
\dot{\mathbf{y}}_{d}=A \mathbf{y}_{d}-F\left(\mathbf{y}_{d}\right)+B u_{d}\left(\mathbf{y}_{d}\right), \quad \mathbf{y}_{d}(0)=\mathbf{y}_{0}
$$


Below we will also derive an estimate for the open-loop control, i.e., the function defined by

$$
u_{d}:[0, \infty) \rightarrow U, t \mapsto u_{d}(t):=u_{d}\left(\mathbf{y}_{d}(t)\right)
$$

which is obtained via closed-loop dynamics here. With slight abuse of notation, the open-loop control $u_{d}(t)$ as well as its closed-loop interpretation $u_{d}\left(\mathbf{y}_{d}(t)\right)$ will both be denoted with $u_{d}$.

We begin with some local Lipschitz continuity estimates for the nonlinear part of the feedback law. For this purpose, we set

$$
G_{k}(\mathbf{y}):=-\frac{1}{\alpha(k-1) !} B B^{*} D^{k} \mathcal{V}(0)(\cdot, \mathbf{y}, \ldots, \mathbf{y}),
$$

for all $k \geq 3$. The closed-loop system can be reformulated as follows:

$$
\begin{aligned}
\dot{\mathbf{y}}_{d} & =A_{\pi} \mathbf{y}_{d}-F\left(\mathbf{y}_{d}\right)-\frac{1}{\alpha} \sum_{k=3}^{d} \frac{1}{(k-1) !} B B^{*} D^{k} \mathcal{V}(0)\left(\cdot, \mathbf{y}_{d}, \ldots, \mathbf{y}_{d}\right) \\
& =A_{\pi} \mathbf{y}_{d}-F\left(\mathbf{y}_{d}\right)+\sum_{k=3}^{d} G_{k}\left(\mathbf{y}_{d}\right)
\end{aligned}
$$

Lemma 20 For all $k \geq 3$, there exists a constant $C(k)>0$ such that for all $\mathbf{y}$ and $\mathbf{z} \in Y$,

$$
\left\|G_{k}(\mathbf{y})-G_{k}(\mathbf{z})\right\|_{Y} \leq C(k)\|\mathbf{y}-\mathbf{z}\|_{Y} \max \left(\|\mathbf{y}\|_{Y},\|\mathbf{z}\|_{Y}\right)^{k-2}
$$

Moreover, for all $\delta \in[0,1]$, for all $\mathbf{y}$ and $\mathbf{z} \in W_{\infty}$ such that $\|\mathbf{y}\|_{W_{\infty}} \leq \delta$ and $\|\mathbf{z}\|_{W_{\infty}} \leq \delta$

$$
\left\|G_{k}(\mathbf{y})-G_{k}(\mathbf{z})\right\|_{L^{2}\left(0, \infty ; V^{\prime}\right)} \leq C(k) \delta\|\mathbf{y}-\mathbf{z}\|_{W_{\infty}} .
$$

Proof We have the identity

$$
\begin{aligned}
& D^{k} \mathcal{V}(0)(\cdot, \mathbf{y}, \ldots, \mathbf{y})-D^{k} \mathcal{V}(0)(\cdot, \mathbf{z}, \ldots, \mathbf{z})=D^{k} \mathcal{V}(0)(\cdot, \mathbf{y}-\mathbf{z}, \mathbf{y}, \ldots, \mathbf{y}) \\
& +D^{k} \mathcal{V}(0)(\cdot, \mathbf{z}, \mathbf{y}-\mathbf{z}, \ldots, \mathbf{y})+\cdots+D^{k} \mathcal{V}(0)(\cdot, \mathbf{z}, \ldots, \mathbf{z}, \mathbf{y}-\mathbf{z})
\end{aligned}
$$

The first inequality easily follows, with $C(k)=\frac{1}{\alpha(k-2) !}\|B\|_{\mathcal{L}(U, Y)}^{2}\left\|D^{k} \mathcal{V}(0)\right\|$ and $\left\|D^{k} \mathcal{V}(0)\right\|$ as defined in (5). We also obtain that for all $\mathbf{y}$ and $\mathbf{z} \in W_{\infty}$,

$$
\left\|G_{k}(\mathbf{y})-G_{k}(\mathbf{z})\right\|_{L^{2}\left(0, \infty ; V^{\prime}\right)} \leq C(k)\|\mathbf{y}-\mathbf{z}\|_{W_{\infty}} \max \left(\|\mathbf{y}\|_{W_{\infty}},\|\mathbf{z}\|_{W_{\infty}}\right)^{k-2} .
$$

The second inequality follows, since $k \geq 3$ and $\delta \leq 1$. 
The well-posedness of the closed-loop system can be now established with the same tools as those used in Lemma 5.

Theorem 21 Let $d \geq 2$. Let $C$ and $C(k)$ denote the constants from Lemmas 4 and 20. There exists a constant $M_{\mathrm{cls}}$ such that for all $\mathbf{y}_{0} \in Y$ with

$$
\left\|\mathbf{y}_{0}\right\|_{Y} \leq \frac{1}{4\left(C+\sum_{k=3}^{d} C(k)\right) M_{\mathrm{cls}}^{2}}
$$

the closed-loop system (51) has a unique solution $\mathbf{y}_{d}$ in $W_{\infty}$, which satisfies

$$
\left\|\mathbf{y}_{d}\right\|_{W_{\infty}} \leq 2 M_{\mathrm{cls}}\left\|\mathbf{y}_{0}\right\|_{Y}
$$

Proof The existence of a solution $\mathbf{y} \in W_{\infty}$, satisfying (55), can be obtained exactly as in Lemma 5. Thus we only discuss uniqueness. Let $\mathbf{y}$ and $\mathbf{z}$ denote two solutions to (51) in $W_{\infty}$. Let us set $\mathbf{e}=\mathbf{y}-\mathbf{z}$. Arguing as in the proof of Lemma 5, one can prove the existence of $M>0$ such that

$$
\begin{aligned}
\frac{1}{2} \frac{\mathrm{d}}{\mathrm{d} t}\|\mathbf{e}\|_{Y}^{2} \leq & M\left(1+\|\mathbf{y}\|_{Y}^{2}\|\mathbf{y}\|_{V}^{2}+\|\mathbf{z}\|_{Y}\left\|^{2} \mathbf{z}\right\|_{V}^{2}\right. \\
& \left.+\sum_{k=3}^{d} C(k)^{2} \max \left(\|\mathbf{y}\|_{Y},\|\mathbf{z}\|_{Y}\right)^{2(k-2)}\right)\|\mathbf{e}\|_{Y}^{2},
\end{aligned}
$$

for all $t \geq 0$. Since $\mathbf{y}$ and $\mathbf{z} \in W_{\infty}$ and $\mathbf{e}(0)=0$, we obtain with Gronwall's inequality that $\mathbf{e}=0$, which proves the uniqueness of the solution to the closed-loop system.

Theorem 22 Let $d \geq 2$. There exist $\delta_{6}>0$ and $M>0$ such that for all $\mathbf{y}_{0} \in B_{Y}\left(\delta_{6}\right)$, it holds that

$$
\begin{aligned}
\left\|\overline{\mathbf{y}}-\mathbf{y}_{d}\right\|_{W_{\infty}} & \leq M\left\|\mathbf{y}_{0}\right\|_{Y}^{d}, \\
\max \left(\left\|\bar{u}-u_{d}\right\|_{L^{2}(0, \infty ; U)},\left\|\bar{u}-u_{d}\right\|_{L^{\infty}(0, \infty ; U)}\right) & \leq M\left\|\mathbf{y}_{0}\right\|_{Y}^{d},
\end{aligned}
$$

where $(\overline{\mathbf{y}}, \bar{u})=\left(\mathcal{Y}\left(\mathbf{y}_{0}\right), \mathcal{U}\left(\mathbf{y}_{0}\right)\right), \mathbf{y}_{d}$ is the solution of the closed-loop system (51) with initial condition $\mathbf{y}_{0}$, and $u_{d}$ is as defined in (52).

Proof Let us fix $\delta_{6}=\min \left(\delta_{5},\left(4\left(C+\sum_{k=3}^{d} C(k)\right) M_{\mathrm{cls}}^{2}\right)^{-1}\right)$, so that Proposition 16 and Theorem 21 apply for $\mathbf{y}_{0} \in B_{Y}\left(\delta_{6}\right)$. By Taylor's theorem, see, e.g., [43, Theorem 4A], there exists $\delta>0$ such that for all $\mathbf{y} \in B_{Y}(\delta)$,

$$
D \mathcal{V}(\mathbf{y})=\sum_{k=2}^{d} \frac{1}{(k-1) !} D^{k} \mathcal{V}(0)(\cdot, \mathbf{y}, \ldots, \mathbf{y})+R_{d}(\mathbf{y}),
$$

where the remainder term $R_{d}$ satisfies

$$
\left\|R_{d}(\mathbf{y})\right\|_{Y} \leq M\|\mathbf{y}\|_{Y}^{d}
$$


for some constant $M$ independent of $\mathbf{y}$. Reducing if necessary $\delta_{6}$, we have that $\|\overline{\mathbf{y}}(t)\|_{Y} \leq \delta$ for all $t \geq 0$. Combining then (43) and the Taylor expansion (56), we obtain that

$$
\dot{\overline{\mathbf{y}}}=A \overline{\mathbf{y}}-F(\overline{\mathbf{y}})-\frac{1}{\alpha} B B^{*} D \mathcal{V}(\overline{\mathbf{y}})=A_{\pi} \overline{\mathbf{y}}-F(\overline{\mathbf{y}})+\sum_{k=3}^{d} G_{k}(\overline{\mathbf{y}})-\frac{1}{\alpha} B B^{*} R_{d}(\overline{\mathbf{y}}) .
$$

Let us now consider the error dynamics $\mathbf{e}:=\overline{\mathbf{y}}-\mathbf{y}_{d}$. We have $\mathbf{e}(0)=0$, moreover by (54) and (57),

$$
\dot{\mathbf{e}}=A_{\pi} \mathbf{e}-F(\overline{\mathbf{y}})+F\left(\mathbf{y}_{d}\right)+\sum_{k=3}^{d}\left(G_{k}(\overline{\mathbf{y}})-G_{k}\left(\mathbf{y}_{d}\right)\right)-\frac{1}{\alpha} B B^{*} R_{d}(\overline{\mathbf{y}})
$$

Alternatively, e can be expressed as the solution of the system

$$
\dot{\mathbf{e}}=A_{\pi} \mathbf{e}+\mathbf{f}, \quad \mathbf{e}(0)=0,
$$

where the source term $\mathbf{f}$ is given by

$$
\mathbf{f}=-F(\overline{\mathbf{y}})+F\left(\mathbf{y}_{d}\right)+\sum_{k=3}^{d}\left(G_{k}(\overline{\mathbf{y}})-G_{k}\left(\mathbf{y}_{d}\right)\right)-\frac{1}{\alpha} B B^{*} R_{d}(\overline{\mathbf{y}}) .
$$

Consider $\tilde{\delta} \in(0,1]$. The precise value of $\tilde{\delta}$ will be fixed later. By Lemma 12 and Theorem 21, we can reduce $\delta_{6}$ so that $\max \left(\|\overline{\mathbf{y}}\|_{W_{\infty}},\left\|\mathbf{y}_{d}\right\|_{W_{\infty}}\right) \leq \tilde{\delta}$. We first observe that

$$
\left\|\frac{1}{\alpha} B B^{*} R_{d}(\overline{\mathbf{y}})\right\|_{L^{2}\left(0, \infty ; V^{\prime}\right)} \leq M\|\overline{\mathbf{y}}\|_{L^{\infty}(0, \infty ; Y)}^{d-1}\|\overline{\mathbf{y}}\|_{L^{2}(0, \infty ; Y)} \leq M\left\|\mathbf{y}_{0}\right\|_{Y}^{d} .
$$

Applying further Lemmas 4 and 20, we obtain

$$
\begin{aligned}
\|\mathbf{f}\|_{L^{2}\left(0, \infty ; V^{\prime}\right)} \leq & M\left(\left\|F(\overline{\mathbf{y}})-F\left(\mathbf{y}_{d}\right)\right\|_{L^{2}\left(0, \infty ; V^{\prime}\right)}\right. \\
& \left.+\sum_{k=3}^{d}\left\|G_{k}(\overline{\mathbf{y}})-G_{k}\left(\mathbf{y}_{d}\right)\right\|_{L^{2}\left(0, \infty ; V^{\prime}\right)}+\left\|\mathbf{y}_{0}\right\|_{Y}^{d}\right) \\
\leq & M\left(\tilde{\delta}\|\mathbf{e}\|_{W_{\infty}}+\left\|\mathbf{y}_{0}\right\|_{Y}^{d}\right) .
\end{aligned}
$$

For the solution of system (58) we thus obtain the estimate

$$
\|\mathbf{e}\|_{W_{\infty}} \leq M\|\mathbf{f}\|_{L^{2}\left(0, \infty ; V^{\prime}\right)} \leq M\left(\tilde{\delta}\|\mathbf{e}\|_{W_{\infty}}+\left\|\mathbf{y}_{0}\right\|_{Y}^{d}\right) .
$$

The constant $M>0$ in the above estimate is independent of $\tilde{\delta}$. We can now define $\tilde{\delta}=\min \left(1, \frac{1}{2 M}\right)$. The first estimate on $\left\|\overline{\mathbf{y}}-\mathbf{y}_{d}\right\|_{W_{\infty}}$ follows. 
Let us estimate $\bar{u}-u_{d}$. By (43) and by definition of the generated open-loop control $u_{d}$, we have that

$$
\bar{u}-u_{d}=-\frac{1}{\alpha} B^{*}\left(D \mathcal{V}(\overline{\mathbf{y}})-D \mathcal{V}_{d}\left(\mathbf{y}_{d}\right)\right)=-\frac{1}{\alpha} B^{*}\left(R_{d}(\overline{\mathbf{y}})+D \mathcal{V}_{d}(\overline{\mathbf{y}})-D \mathcal{V}_{d}\left(\mathbf{y}_{d}\right)\right)
$$

Let us estimate the two terms of the right-hand side. It is easy to check that

$$
\max \left(\left\|R_{d}(\overline{\mathbf{y}})\right\|_{L^{\infty}(0, \infty ; Y)},\left\|R_{d}(\overline{\mathbf{y}})\right\|_{L^{2}(0, \infty ; Y)}\right) \leq M\left\|\mathbf{y}_{0}\right\|_{Y}^{d} .
$$

Using the techniques of Lemma 20 and the estimate on $\left\|\overline{\mathbf{y}}-\mathbf{y}_{d}\right\|_{W_{\infty}}$, we also obtain that

$$
\begin{aligned}
& \max \left(\left\|D \mathcal{V}_{d}(\overline{\mathbf{y}})-D \mathcal{V}_{d}\left(\mathbf{y}_{d}\right)\right\|_{L^{2}(0, \infty ; Y)},\left\|D \mathcal{V}_{d}(\overline{\mathbf{y}})-D \mathcal{V}_{d}\left(\mathbf{y}_{d}\right)\right\|_{L^{\infty}(0, \infty ; Y)}\right) \\
& \quad \leq M\left\|\overline{\mathbf{y}}-\mathbf{y}_{d}\right\|_{W_{\infty}} \leq M\left\|\mathbf{y}_{0}\right\|_{Y}^{d} .
\end{aligned}
$$

The second estimate on $\bar{u}-u_{d}$ follows.

\subsection{Estimates for the Pressure}

It is well-known that for $\mathbf{y}_{0} \in Y$, the pressure term that can be associated to the Navier-Stokes equations is a distribution only (see, e.g., [39], [40, Chapter III-§3]). In the following, we redemonstrate this fact and we argue that a result analogous to Theorem 22 also holds for the pressure, provided the latter is considered in $W^{-1, \infty}\left(0, \infty ; L_{0}^{2}(\Omega)\right)=W_{0}^{1,1}\left(0, \infty ; L_{0}^{2}(\Omega)\right)^{\prime}$ with

$$
W_{0}^{1,1}\left(0, \infty ; L_{0}^{2}(\Omega)\right)=\left\{v \in W^{1,1}\left(0, \infty ; L_{0}^{2}(\Omega)\right) \mid v(0)=0\right\}
$$

and

$$
L_{0}^{2}(\Omega)=\left\{v \in L^{2}(\Omega) \mid \int_{\Omega} v(x) \mathrm{d} x=0\right\} .
$$

We define similarly $W_{0}^{1,1}\left(0, \infty ; \mathbb{H}_{0}^{1}(\Omega)\right)$. We recall here that $W_{0}^{1,1}\left(0, \infty ; \mathbb{H}_{0}^{1}(\Omega)\right)$ embeds continuously into $L^{\infty}\left(0, \infty ; \mathbb{H}_{0}^{1}(\Omega)\right) \cap L^{2}\left(0, \infty ; \mathbb{H}_{0}^{1}(\Omega)\right)$. Further the elements $\boldsymbol{\phi}$ of $W_{0}^{1,1}\left(0, \infty ; \mathbb{H}_{0}^{1}(\Omega)\right)$ can be identified a.e. with continuous functions on $[0, \infty)$ and satisfy $\lim _{t \rightarrow \infty}\|\phi(t)\|_{\mathbb{H}_{0}^{1}(\Omega)}=0$. We use the properties of Banach-space valued functions as summarized in [14, Chapter II-§5].

Lemma 23 Let $(\mathbf{y}, u) \in W_{\infty} \times L^{2}(0, \infty ; U)$ be such that $\dot{\mathbf{y}}=A \mathbf{y}-F(\mathbf{y})+B u$. Then, there exists a unique $p \in W^{-1, \infty}\left(0, \infty ; L_{0}^{2}(\Omega)\right)$ such that

$$
\dot{\mathbf{y}}=A \mathbf{y}-F(\mathbf{y})+B u-\nabla p \quad \text { in } W^{1,1}\left(0, \infty ; \mathbb{H}_{0}^{1}(\Omega)\right)^{\prime}
$$


that is,

$$
\begin{aligned}
-\int_{0}^{\infty}\langle\mathbf{y}(t), \dot{\boldsymbol{\phi}}(t)\rangle_{Y} \mathrm{~d} t= & \int_{0}^{\infty}\langle A \mathbf{y}(t)-F(\mathbf{y}(t))+B u(t), \boldsymbol{\phi}(t)\rangle_{\mathbb{H}^{-1}(\Omega), \mathbb{H}_{0}^{1}(\Omega)} \mathrm{d} t \\
& +\langle p, \operatorname{div} \boldsymbol{\phi}\rangle_{W^{-1, \infty}\left(0, \infty, L_{0}^{2}(\Omega)\right), W_{0}^{1,1}\left(0, \infty ; L_{0}^{2}(\Omega)\right)}
\end{aligned}
$$

for all $\phi \in W_{0}^{1,1}\left(0, \infty ; \mathbb{H}_{0}^{1}(\Omega)\right)$. Moreover,

$$
\|p\|_{W^{-1, \infty}\left(0, \infty, L_{0}^{2}(\Omega)\right)} \leq M\left(\|\mathbf{y}\|_{W_{\infty}}+\|\mathbf{y}\|_{W_{\infty}}^{2}+\|u\|_{L^{2}(0, \infty ; U)}\right)
$$

for a constant $M$ independent of $(\mathbf{y}, u)$.

Proof We follow the technique consisting in integrating the state equation, see, e.g., [14, Chapter V-§1] and introduce

$$
\mathbf{G}(t)=\mathbf{y}(t)-\mathbf{y}_{0}+\int_{0}^{t} \mathbf{g}(s) \mathrm{d} s, \quad \text { with: } \mathbf{g}(s)=A \mathbf{y}(s)-F(\mathbf{y}(s))+B u(s) .
$$

It can be easily shown that $\mathbf{g} \in L^{2}\left(0, \infty ; \mathbb{H}^{-1}(\Omega)\right)$ and that there exists a constant $M>0$ independent of $(\mathbf{y}, u)$ such that

$$
\|\mathbf{g}\|_{L^{2}\left(0, \infty ; \mathbb{H}^{-1}(\Omega)\right)} \leq M\left(\|\mathbf{y}\|_{W_{\infty}}+\|\mathbf{y}\|_{W_{\infty}}^{2}+\|u\|_{L^{2}(0, \infty ; U)}\right) .
$$

This estimate can be obtained with the Cauchy-Schwarz inequality and Proposition 1(i), which also holds true in $\mathbb{H}^{-1}(\Omega)$ (in place of $V^{\prime}$ ). Since $\mathbf{y} \in W_{\infty}$, it further follows that $\mathbf{G}$ is a continuous function of time with values in $\mathbb{H}^{-1}(\Omega)$. Moreover, $\langle\mathbf{G}(t), \boldsymbol{\psi}\rangle_{\mathbb{H}^{-1}(\Omega), \mathbb{H}_{0}^{1}(\Omega)}=0$ for all $t \in[0, \infty)$ and $\psi \in V$. Hence for all $t \in[0, \infty)$, there exists a unique $\mathcal{P}(t) \in L_{0}^{2}(\Omega)$ such that $\mathbf{G}(t)=-\nabla \mathcal{P}(t)$, see, e.g., [14, Theorem IV.2.3]. Let us prove that $\mathcal{P} \in C\left([0, \infty), L_{0}^{2}(\Omega)\right)$. Recall first that there exists an operator $\mathcal{K} \in \mathcal{L}\left(L_{0}^{2}(\Omega), \mathbb{H}_{0}^{1}(\Omega)\right)$ with the property that

$$
\operatorname{div}(\mathcal{K} \rho)=\rho, \quad \forall \rho \in L_{0}^{2}(\Omega)
$$

see [14, Theorem IV.3.1]. Let $\rho \in L_{0}^{2}(\Omega)$ be arbitrary and let $\phi=\mathcal{K} \rho$. For all $t$ and $\tau$ in $[0, \infty)$, we have

$$
\begin{aligned}
\langle\mathcal{P}(t)-\mathcal{P}(\tau), \rho\rangle_{L_{0}^{2}(\Omega)} & =-\langle\nabla \mathcal{P}(t)-\nabla \mathcal{P}(\tau), \boldsymbol{\phi}\rangle_{\mathbb{H}^{-1}(\Omega), \mathbb{H}_{0}^{1}(\Omega)} \\
& =\langle\mathbf{G}(t)-\mathbf{G}(\tau), \boldsymbol{\phi}\rangle_{\mathbb{H}^{-1}(\Omega), \mathbb{H}_{0}^{1}(\Omega)} \\
& \leq\|\mathcal{K}\|_{\mathcal{L}\left(L_{0}^{2}(\Omega), \mathbb{H}_{0}^{1}(\Omega)\right)}\|\mathbf{G}(t)-\mathbf{G}(\tau)\|_{\mathbb{H}^{-1}(\Omega)}\|\rho\|_{L_{0}^{2}(\Omega)}
\end{aligned}
$$

It follows that $\|\mathcal{P}(t)-\mathcal{P}(\tau)\|_{L_{0}^{2}(\Omega)} \leq M\|\mathbf{G}(t)-\mathbf{G}(\tau)\|_{\mathbb{H}^{-1}(\Omega)}$, which concludes the proof of continuity of $\mathcal{P}$. We now introduce the distributional derivative $p=\frac{\mathrm{d}}{\mathrm{d} t} \mathcal{P}$ and 
establish that $p \in W^{-1, \infty}\left(0, \infty ; L_{0}^{2}(\Omega)\right)$. Let $\rho \in \mathcal{C}_{c}^{\infty}\left(0, \infty ; L_{0}^{2}(\Omega)\right)$ be arbitrary and set $\boldsymbol{\phi}(t)=\mathcal{K} \rho(t)$. Note that $\boldsymbol{\phi} \in \mathcal{C}_{c}^{\infty}\left(0, \infty ; \mathbb{H}_{0}^{1}(\Omega)\right)$. We have

$$
\begin{aligned}
\langle p, \rho\rangle & =-\int_{0}^{\infty}\langle\mathcal{P}(t), \dot{\rho}(t)\rangle_{L_{0}^{2}(\Omega)} \mathrm{d} t=-\int_{0}^{\infty}\langle\mathcal{P}(t), \operatorname{div} \dot{\boldsymbol{\phi}}(t)\rangle_{L_{0}^{2}(\Omega)} \mathrm{d} t \\
& =\int_{0}^{\infty}\langle\nabla \mathcal{P}(t), \dot{\boldsymbol{\phi}}(t)\rangle_{\mathbb{H}^{-1}(\Omega), \mathbb{H}_{0}^{1}(\Omega)} \mathrm{d} t \\
& =-\int_{0}^{\infty}\left\langle\mathbf{y}(t)-\mathbf{y}_{0}-\int_{0}^{t} \mathbf{g}(s) \mathrm{d} s, \dot{\boldsymbol{\phi}}(t)\right\rangle_{\mathbb{H}^{-1}(\Omega), \mathbb{H}_{0}^{1}(\Omega)} \mathrm{d} t \\
& =-\int_{0}^{\infty}\left\langle\mathbf{y}(t)-\mathbf{y}_{0}, \dot{\boldsymbol{\phi}}(t)\right\rangle_{\mathbb{H}^{-1}(\Omega), \mathbb{H}_{0}^{1}(\Omega)}+\langle\mathbf{g}(t), \boldsymbol{\phi}(t)\rangle_{\mathbb{H}^{-1}(\Omega), \mathbb{H}_{0}^{1}(\Omega)} \mathrm{d} t
\end{aligned}
$$

Recalling the embedding of $W_{0}^{1,1}\left(0, \infty ; \mathbb{H}_{0}^{1}(\Omega)\right)$ in $L^{2}\left(0, \infty ; \mathbb{H}_{0}^{1}(\Omega)\right)$, we deduce that

$$
\langle p, \rho\rangle \leq M\left(\|\mathbf{y}\|_{L^{\infty}\left(0, \infty ; \mathbb{H}^{-1}(\Omega)\right)}+\|\mathbf{g}\|_{L^{2}\left(0, \infty ; \mathbb{H}^{-1}(\Omega)\right)}\right)\|\boldsymbol{\phi}\|_{W_{0}^{1,1}\left(0, \infty ; \mathbb{H}_{0}^{1}(\Omega)\right)} .
$$

Using then estimate (62), we obtain that $p$ can be extended to an element of $W^{-1, \infty}\left(0, \infty ; L_{0}^{2}(\Omega)\right)$ satisfying estimate $(60)$.

With the same calculations as above, we can show that for all $\phi \in W_{0}^{1,1}\left(0, \infty ; \mathbb{H}_{0}^{1}(\Omega)\right)$,

$$
\langle p, \operatorname{div} \boldsymbol{\phi}\rangle=-\int_{0}^{\infty}\langle\mathbf{y}(t), \dot{\boldsymbol{\phi}}(t)\rangle_{\mathbb{H}^{-1}(\Omega), \mathbb{H}_{0}^{1}(\Omega)}+\langle\mathbf{g}(t), \boldsymbol{\phi}(t)\rangle_{\mathbb{H}^{-1}(\Omega), \mathbb{H}_{0}^{1}(\Omega)} \mathrm{d} t,
$$

which proves that $p$ satisfies (59). Let us prove the uniqueness. Let $\tilde{p} \in$ $W^{-1, \infty}\left(0, \infty ; L_{0}^{2}(\Omega)\right)$ satisfy $(59)$. Let $\rho \in W_{0}^{1,1}\left(0, \infty ; L_{0}^{2}(\Omega)\right)$ be arbitrary and let us set $\boldsymbol{\phi}=\mathcal{K} \rho$. Then, by (59), we have

$$
\begin{aligned}
0 & =\langle p-\tilde{p}, \operatorname{div} \boldsymbol{\phi}\rangle_{W^{-1, \infty}\left(0, \infty ; L_{0}^{2}(\Omega)\right), W_{0}^{1,1}\left(0, \infty ; L_{0}^{2}(\Omega)\right)} \\
& =\langle p-\tilde{p}, \rho\rangle_{W^{-1, \infty}\left(0, \infty ; L_{0}^{2}(\Omega), W_{0}^{1,1}\left(0, \infty ; L_{0}^{2}(\Omega)\right)\right.}
\end{aligned}
$$

which proves that $p=\tilde{p}$ and concludes the proof.

We have the following result, extending Theorem 22 .

Proposition 24 Let $d \geq 2$. There exists $M>0$ such that for all $\mathbf{y}_{0} \in Y$ with $\left\|\mathbf{y}_{0}\right\|_{Y} \leq$ $\delta_{6}$,

$$
\left\|\bar{p}-p_{d}\right\|_{W^{-1, \infty}\left(L_{0}^{2}\right)} \leq M\left\|\mathbf{y}_{0}\right\|_{Y}^{d},
$$

where $\bar{p}$ and $p_{d}$ denote the pressure terms associated with $(\overline{\mathbf{y}}, \bar{u})$ and $\left(\mathbf{y}_{d}, u_{d}\right)$ respectively.

Proof We have introduced in the proof of Lemma 23 the term $\mathbf{g}$ associated with a feasible pair $(\mathbf{y}, u)$. Let us denote by $\overline{\mathbf{g}}$ and $\mathbf{g}_{d}$ the corresponding terms associated 


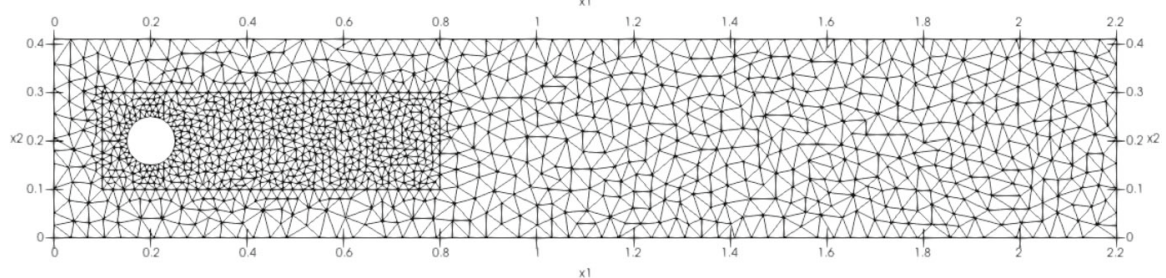

Fig. 1 Geometry and non uniform grid

with $(\overline{\mathbf{y}}, \bar{u})$ and $\left(\mathbf{y}_{d}, u_{d}\right)$. One can verify that as a consequence of Theorem $22, \| \overline{\mathbf{g}}-$ $\mathbf{g}_{d}\left\|_{L^{2}\left(0, \infty ; \mathbb{H}^{-1}(\Omega)\right)} \leq M\right\| \mathbf{y}_{0} \|_{Y}^{d}$. Proposition 24 follows then with similar calculations to those performed in the proof of Lemma 23.

\section{A Numerical Example}

In this section, we present numerical simulations for the two-dimensional NavierStokes equations and computed feedback laws of order 2 and 3. The discretization procedure and the example setups are classical and are taken from [9]. The main purpose is to show that the computation of higher order feedback laws is possible and, depending on the chosen parameters, visible differences to a Riccati-based feedback law can be observed.

\subsection{Setup and Discretization}

We briefly summarize the numerical implementation provided in [9]. Therein a TaylorHood $P_{2}-P_{1}$ finite element discretization for a two dimensional wake behind a cylinder is discussed. The computational domain $\Omega=(0,2.2) \times(0,0.41)$ as well as a non uniform grid are shown in Fig. 1. For all simulations, we use the Reynolds number $\operatorname{Re}:=\frac{1}{v}=90$ and the parabolic inflow profile discussed in [9]. For the upper and lower end of the geometry, no slip boundary conditions are employed. The outflow is modeled by do nothing boundary conditions on the right end of the geometry. For the desired stabilization, we utilize a distributed, separable control acting in the control domain $\Omega_{c}:=[0.27,0.32] \times[0.15,0.25]$. In particular, the control operator is of the form

$$
B u=\sum_{\ell=1}^{3}\left[\begin{array}{c}
0 \\
w_{\ell}\left(x_{2}\right)
\end{array}\right] u_{\ell}(t)+\left[\begin{array}{c}
w_{\ell}\left(x_{2}\right) \\
0
\end{array}\right] u_{\ell+3}(t)
$$

where the control shape functions $w_{1}, w_{2}$ and $w_{3}$ are piecewise linear functions which are constant along the $x_{1}$-direction.

The finite element discretization is computed in FEniCS and the resulting matrices associated with the spatial semidiscretization are exported to MATLAB. As described in detail in [9], the (spatially) discrete system takes the form 


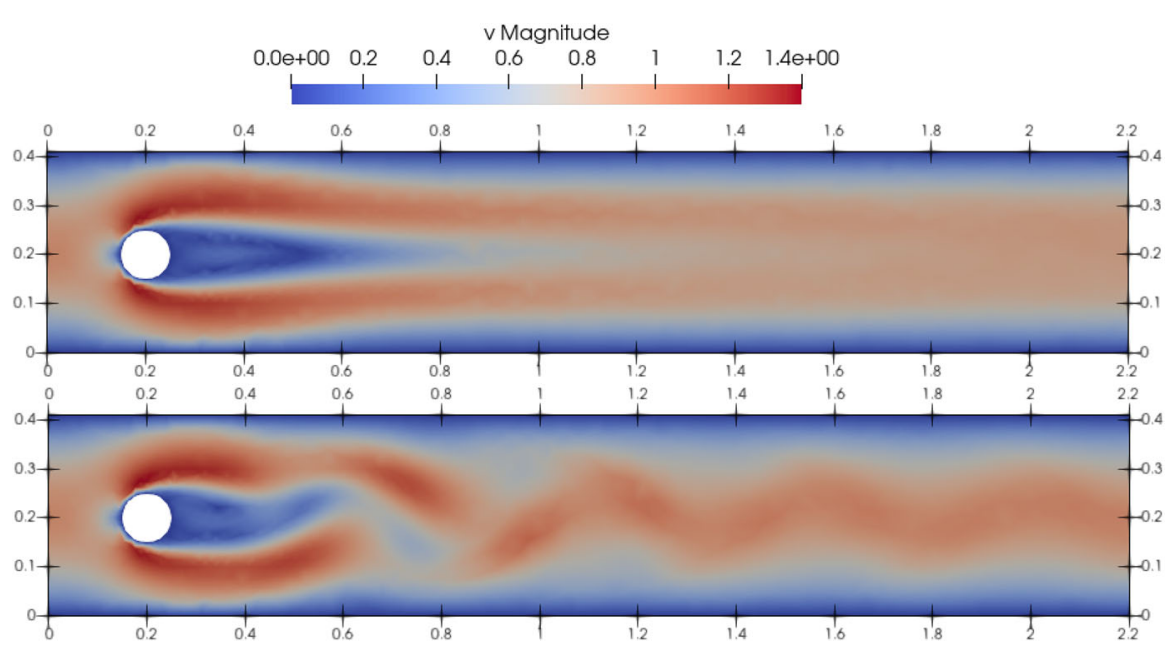

Fig. 2 The steady state and a snapshot of the transient flow regime

$$
\begin{aligned}
E \dot{z}(t) & =-K z(t)+H(z(t) \otimes z(t))+B u(t)+G q(t)+f_{z}, \\
0 & =G^{T} z(t)+f_{q},
\end{aligned}
$$

where $E, K \in \mathbb{R}^{n_{v} \times n_{v}}$ are the mass and stiffness matrices, $G^{T} \in \mathbb{R}^{n_{p} \times n_{v}}$ represents the discrete divergence operator, the tensor matricization $H \in \mathbb{R}^{n_{v} \times n_{v}^{2}}$ represents the trilinear form (9) and $B \in \mathbb{R}^{n_{v} \times 6}$ is the discrete control operator. Note that $H$ can be constructed in such a way that $H\left(z_{1} \otimes z_{2}\right)=H\left(z_{2} \otimes z_{1}\right)$ for any $z_{1}, z_{2} \in \mathbb{R}^{n_{v}}$. The time invariant vectors $f_{z} \in \mathbb{R}^{n_{v}}$ and $f_{q} \in \mathbb{R}^{n_{p}}$ are due to the elimination of the boundary nodes. The following results correspond to a discretization level with $n_{v}=9356$ and $n_{p}=1289$. The velocity profile of the unstable steady state solution $\bar{z}$ shown in Fig. 2 is obtained by a Picard iteration applied to the uncontrolled stationary system, i.e., system (63) with $\dot{z}(t)=0$ and $u(t)=0$. To illustrate that the controller stabilizes this steady state solution, we start the transient simulations of the closed-loop systems from the slightly randomly perturbed steady state $z(0)=\bar{z}+\frac{\|\bar{z}\|_{2}}{2000} \cdot \operatorname{randn}\left(n_{v}, 1\right)$.

\subsection{Reformulation as an ODE System}

System (63) is a system of differential-algebraic equations (DAEs) and hence the results from above are not readily applicable. While a thorough analysis in the framework of control of DAEs is certainly of interest, at this point we employ a reformulation initially proposed in [28] that allows to rewrite the dynamics as a set of ODEs for the velocity vector $z$. As in (3), we consider the shifted variables $y=z-\bar{z}$ and $p=q-\bar{q}$, respectively. Consequently, we obtain

$$
\begin{aligned}
E \dot{y}(t) & =A y(t)+H(y(t) \otimes y(t))+B u(t)+G p(t), \\
0 & =G^{T} y(t),
\end{aligned}
$$


where $A=-K+H(\bar{z} \otimes I+I \otimes \bar{z})$. Let us note that the second equation implies $G^{T} \dot{y}(t)=0$. Following [28, Section 3], from the first equation, we thus obtain

$$
0=G^{T} \dot{y}(t)=G^{T} E^{-1}(A y(t)+H(y(t) \otimes y(t))+B u(t)+G p(t)) .
$$

We can now eliminate the pressure from (64) using the relation

$$
p(t)=-\left(G^{T} E^{-1} G\right)^{-1} G^{T} E^{-1}(A y(t)+H(y(t) \otimes y(t))+B u(t)) .
$$

With the notation $P=I-G\left(G^{T} E^{-1} G\right)^{-1} G^{T} E^{-1}$ this yields the system

$$
E \dot{y}(t)=P A y(t)+P H(y(t) \otimes y(t))+P B u(t) .
$$

In fact, as has been discussed in [11], the matrix $P=P^{2}$ as a discrete realization of the Leray projector. Since $G^{T} y=0$, we have $P^{T} y(t)=y(t)$ so that we can multiply the last equation by $P$ to obtain

$$
\left(P E P^{T}\right) \dot{y}(t)=\left(P A P^{T}\right) y(t)+\left(P H P^{T} \otimes P^{T}\right)(y(t) \otimes y(t))+(P B) u(t) .
$$

Finally, by means of a decomposition $P=\Theta_{\ell} \Theta_{r}^{T}$ with $\Theta_{\ell}^{T} \Theta_{r}=I$ we can project onto the $n_{v}-n_{p}$ dimensional subspace range $(P)$ and arrive at the ODE system

$$
\underbrace{\left(\Theta_{r}^{T} E \Theta_{r}\right)}_{\widetilde{E}} \dot{\tilde{y}}(t)=\underbrace{\left(\Theta_{r}^{T} A \Theta_{r}\right)}_{\widetilde{A}} \tilde{y}(t)+\underbrace{\left(\Theta_{r}^{T} H \Theta_{r} \otimes \Theta_{r}\right)}_{\widetilde{H}} \tilde{y}(t) \otimes \tilde{y}(t)+\underbrace{\left(\Theta_{r}^{T} B\right)}_{\widetilde{B}} u(t),
$$

where $\tilde{y}=\Theta_{\ell}^{T} y(t)$. For the initialization, we use $\tilde{y}(0)=\Theta_{\ell}^{T} y_{0}$. At this point, we emphasize that the explicit formulas yield dense matrices and thus are rather a theoretical tool. In particular, an explicit computation of $\widetilde{H}$ is infeasible for the problem dimension considered here. As a remedy, we work with an implementation that applies the above operations whenever a matrix vector multiplication is needed.

\subsection{Computing the Feedback Gain}

With the previous considerations in mind, we focus on the stabilization problem

$$
\inf _{u \in L^{2}\left(0, \infty ; \mathbb{R}^{6}\right)} J\left(\tilde{y}_{0}, u\right), \quad \text { subject to: } e\left(\tilde{y}_{u}, u\right)=\left(0, \tilde{y}_{0}\right)
$$

where

$$
\begin{aligned}
& J\left(\tilde{y}_{u}, u\right)=\frac{1}{2} \int_{0}^{\infty}\left\|\Theta_{r} \tilde{y}_{u}(t)\right\|_{\mathbb{R}^{n_{v}}}^{2} \mathrm{~d} t+\frac{\alpha}{2} \int_{0}^{\infty}\|u(t)\|_{\mathbb{R}^{6}}^{2} \mathrm{~d} t \\
& e\left(\tilde{y}_{u}, u\right)=\left(\widetilde{E} \dot{\tilde{y}}_{u}-\left(\widetilde{A} \tilde{y}_{u}+\widetilde{H}\left(\tilde{y}_{u} \otimes \tilde{y}_{u}\right)+\widetilde{B} u\right), \tilde{y}(0)\right) .
\end{aligned}
$$


We illustrate the effect of higher order feedback laws by computing the first two non trivial derivatives $D^{2} \mathcal{V}(0)$ and $D^{3} \mathcal{V}(0)$, respectively. For the computation of $D^{2} \mathcal{V}(0) \equiv \Pi \in \mathbb{R}^{\left(n_{v}-n_{p}\right) \times\left(n_{v}-n_{p}\right)}$, we have to solve the algebraic matrix Riccati equation

$$
\widetilde{A}^{T} \Pi \widetilde{E}+\widetilde{E}^{T} \Pi \widetilde{A}-\widetilde{E}^{T} \Pi \widetilde{B} \widetilde{B}^{T} \Pi \widetilde{E}+\Theta_{r}^{T} \Theta_{r}=0
$$

which in our case was done by means of the MATLAB function care. For the third order tensor $D^{3} \mathcal{V}(0) \equiv \mathcal{X} \in \mathbb{R}^{\left(n_{v}-n_{p}\right)^{3}}$ we have to solve a linear system of the form $\mathcal{A}^{T} \mathcal{X}=\mathcal{F}$ where

$$
\begin{aligned}
& \mathcal{A}=\widetilde{E} \otimes \widetilde{E} \otimes \widetilde{A}_{\pi}+\widetilde{E} \otimes \widetilde{A}_{\pi} \otimes \widetilde{E}+\widetilde{A}_{\pi} \otimes \widetilde{E} \otimes \widetilde{E}, \quad \widetilde{A}_{\pi}=\widetilde{A}-\frac{1}{\alpha} \widetilde{B} \widetilde{B}^{T} \Pi \widetilde{E} \\
& \mathcal{F}=-2\left(\widetilde{H}^{T} \otimes \widetilde{E}^{T}+\widetilde{E}^{T} \otimes \widetilde{H}^{T}+\left(I \otimes \mathcal{P}^{T}\right)\left(\widetilde{H}^{T} \otimes \widetilde{E}^{T}\right)\right) \pi
\end{aligned}
$$

where $\pi=\operatorname{vec}(\Pi)$ denotes the vectorization of $\Pi$ and the permutation matrix $\mathcal{P}$ is given by

$$
\mathcal{P}=\left[I \otimes e_{1}, \ldots, I \otimes e_{n_{v}-n_{p}}\right] \in \mathbb{R}^{\left(n_{v}-n_{p}\right)^{2} \times\left(n_{v}-n_{p}\right)^{2}} .
$$

Let us emphasize that $\mathcal{F}$ is the discrete realization of the term $\mathcal{R}_{3}$ in (47). In particular, the tensor $\mathcal{F}$ is symmetric. Note that computing a solution $\mathcal{X}$ to $\mathcal{A}^{T} \mathcal{X}=\mathcal{F}$ is infeasible without using further tools such as model order reduction or tensor calculus as storing the vector $\mathcal{X} \in \mathbb{R}^{\left(n_{v}-n_{p}\right)^{3}}$ already requires more than 4 TB of data. As a remedy, we aim for a direct computation of the corresponding feedback gain

$$
\widetilde{K}=\left(\widetilde{E}^{T} \otimes \widetilde{E}^{T} \otimes \widetilde{B}^{T}\right) \mathcal{X}
$$

without explicitly computing $\mathcal{X}$. With this in mind, we proceed as in [16] and utilize a quadrature-based approximation that has been analyzed in [25]. From [25, Lemma 3], it follows that

$$
\mathcal{A}^{-1}=-\int_{0}^{\infty}\left(e^{t \widetilde{E}^{-1} \widetilde{A}_{\pi}} \widetilde{E}^{-1}\right) \otimes\left(e^{t \widetilde{E}^{-1} \widetilde{A}_{\pi}} \widetilde{E}^{-1}\right) \otimes\left(e^{t \widetilde{E}^{-1} \widetilde{A}_{\pi}} \widetilde{E}^{-1}\right) \mathrm{d} t .
$$

As shown in [25, Theorem 9], the previous integral can be well approximated by a tensor sum of the form

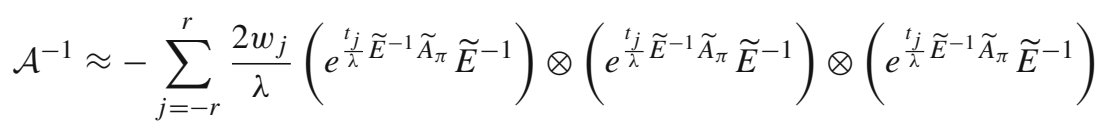

where $t_{j}$ and $w_{j}$ are suitable quadrature points and weights and $\lambda$ denotes a constant determined by the spectrum of the matrix pencil $(\widetilde{E}, \widetilde{A})$. Combining the representation 
in (67), (68) and (69), we obtain the following approximation formula for the feedback gain

$$
\begin{aligned}
\widetilde{K}= & -\sum_{j=-r}^{r} \frac{2 w_{j}}{\lambda}\left(\left(e^{\frac{t_{j}}{\lambda} \widetilde{E}^{-1} \widetilde{A}_{\pi}}\right)^{T}\right) \otimes\left(\left(e^{\frac{t_{j}}{\lambda} \widetilde{E}^{-1} \widetilde{A}_{\pi}}\right)^{T}\right) \otimes\left(\widetilde{B}^{T} \widetilde{E}^{-T}\left(e^{\frac{t_{j}}{\lambda} \widetilde{E}^{-1} \widetilde{A}_{\pi}}\right)^{T}\right) \mathcal{F} \\
= & \sum_{j=-r}^{r} \frac{4 w_{j}}{\lambda}\left(\left(e^{\frac{t_{j}}{\lambda} \widetilde{E}^{-1} \widetilde{A}_{\pi}}\right)^{T}\right) \otimes\left(\left(e^{\frac{t_{j}}{\lambda} \widetilde{E}^{-1} \widetilde{A}_{\pi}}\right)^{T}\right) \otimes\left(\widetilde{B}^{T} \widetilde{E}^{-T}\left(e^{\frac{t_{j}}{\lambda}} \widetilde{E}^{-1} \widetilde{A}_{\pi}\right)^{T}\right) \\
& \times\left(\widetilde{H}^{T} \otimes \widetilde{E}^{T}+\widetilde{E}^{T} \otimes \widetilde{H}^{T}+\left(I \otimes \mathcal{P}^{T}\right)\left(\widetilde{H}^{T} \otimes \widetilde{E}^{T}\right)\right) \pi,
\end{aligned}
$$

with $r=30$ in the numerical examples. By use of algebraic manipulations such as reshaping and transposition of matrices, the computation of the permutation matrix $\mathcal{P}$ as well as computation of the dense matricization $\widetilde{H}$ can be avoided. As a consequence, we obtain an approximation of $\widetilde{K} \in \mathbb{R}^{6\left(n_{v}-n_{p}\right)^{2}}$ whose storage requires less than $4 \mathrm{~GB}$ of data. Let us point out that the above considerations do not fully break the curse of dimensionality but nevertheless allow us to compute a third order feedback law even for a spatially discretized PDE. For the simulation of the time-varying systems, we make use of the MATLAB function ode23 with the standard relative error tolerance $10^{-3}$. In each time step, the control laws $u_{2}(\tilde{y})$ and $u_{3}(\tilde{y})$ are obtained via

$$
\begin{aligned}
& u_{2}(\tilde{y})=-\frac{1}{\alpha} \widetilde{B}^{T} \Pi \tilde{E} \tilde{y}, \\
& u_{3}(\tilde{y})=-\frac{1}{\alpha} \widetilde{B}^{T} \Pi \tilde{E} \tilde{y}-\frac{1}{\alpha}\left(I_{6} \otimes \tilde{y}^{T} \otimes \tilde{y}^{T}\right) \widetilde{K}
\end{aligned}
$$

where $I_{6}$ denotes the identity matrix for the control space $\mathbb{R}^{6}$.

\subsection{Results}

Below, we present a numerical comparison for two different values of $\alpha$. In Fig. 3, the control laws corresponding to (66) with $\alpha=1$ are shown. We observe that both feedback laws $u_{2}$ and $u_{3}$, respectively, exhibit a similar behavior and create vortices which induce the desired control. Indeed, the control velocities in $x_{1}$-direction are of opposite sign (with the centered velocitiy field being negligible) while the control velocities in $x_{2}$-direction all have the same sign.

For $\alpha=10^{-4}$, Fig. 4 shows more visible differences between the control laws.

It would certainly be of interest to investigate the numerical convergence behavior as the order of the control laws increases. At the moment, however this is out of reach, and could be based on model reduction techniques in an independent numerical endeavor. In Fig. 4, we observe that the amplitudes of the $u_{3}$ controls decay more rapidly than those of the $u_{2}$ controls. This is consistent with Fig. 5, where we compare the dynamical behavior of $\left\|u_{2}\right\|_{2}^{2}$ and $\left\|u_{3}\right\|_{2}^{2}$. Let us emphasize that for $\alpha=10^{-4}$, for 

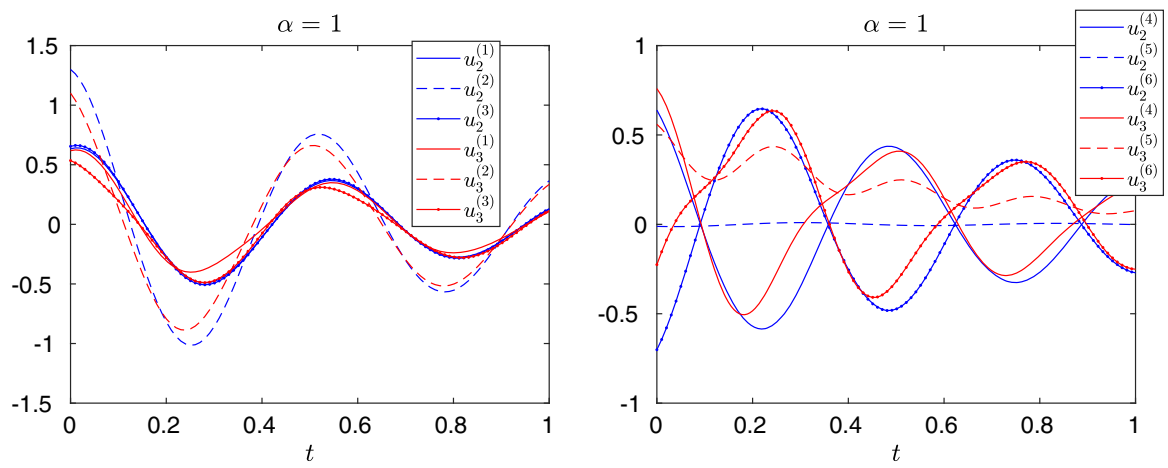

Fig. 3 Control laws in $x_{2}$ (left) and $x_{1}$-direction (right) for $\alpha=1$
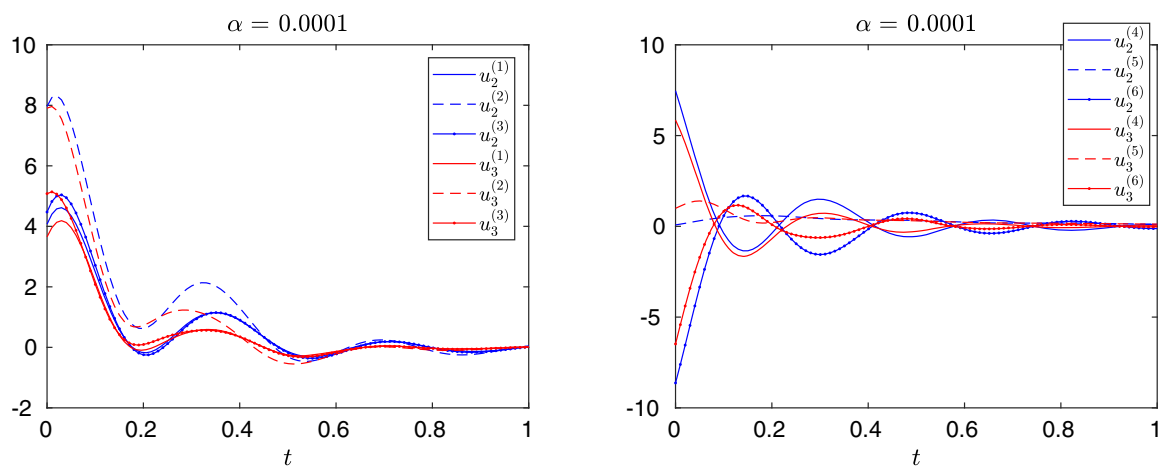

Fig. 4 Control laws in $x_{2}$ (left) and $x_{1}$-direction (right) for $\alpha=10^{-4}$

all $t$, the norm of the control law $u_{3}(t)$ is smaller than the one of $u_{2}(t)$. For the values of the cost functionals, we obtain

$$
\begin{aligned}
& J\left(\tilde{y}_{u_{2}}, u_{2}\right)=0.9546, \quad J\left(\tilde{y}_{u_{3}}, u_{3}\right)=0.8432, \quad \text { for } \alpha=1, \\
& J\left(\tilde{y}_{u_{2}}, u_{2}\right)=0.0128, \quad J\left(\tilde{y}_{u_{3}}, u_{3}\right)=0.0125, \quad \text { for } \alpha=10^{-4},
\end{aligned}
$$

which indicates that higher order feedback laws can be of interest for feedback stabilization.

\section{Outlook}

In the present paper we demonstrated that the approach that we carried out for obtaining Taylor approximations to the value function of optimal control problems related to the Fokker-Planck equation, is also applicable for optimal control of the Navier-Stokes equations in dimension two. The question arises to which extent analogous results can be obtained for dimension three and for boundary control problems. In dimension three the situation will be significantly different from that of the current paper. It will 

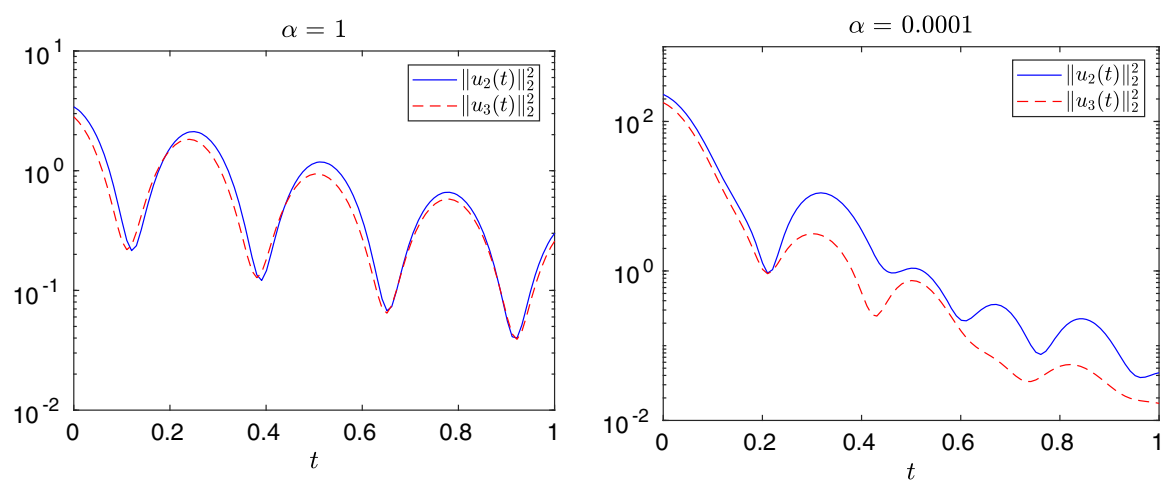

Fig. 5 Dynamical behavior of the control norms for $\alpha=1$ and $\alpha=10^{-4}$

not be possible to work with weak variational solutions. Rather one has to resort to strong variational solutions, and thus one can expect at best that the value function is smooth on $V$ rather than on $Y$. This leads to difficulties for the operator representations of the derivatives of the value function. Alternatively one can start by analyzing (47) as equations for abstract multilinear forms $D^{k} \mathcal{V}(0)$, which are not necessarily obtained of derivatives of $\mathcal{V}$. This is an approach which we plan to follow.

Acknowledgements Open access funding provided by University of Graz. This work was partly supported by the ERC advanced Grant 668998 (OCLOC) under the EU's H2020 research program. The authors would like to thank Jan Heiland for making available his finite element based code for solving the state equation as well as many helpful and interesting discussions on the numerical examples.

Open Access This article is distributed under the terms of the Creative Commons Attribution 4.0 International License (http://creativecommons.org/licenses/by/4.0/), which permits unrestricted use, distribution, and reproduction in any medium, provided you give appropriate credit to the original author(s) and the source, provide a link to the Creative Commons license, and indicate if changes were made.

\section{Appendix}

The appendix is dedicated to the proof of Proposition 15.

We follow the notation from, e.g., [5] and define the following spaces

$$
\begin{aligned}
V_{n}^{s} & :=\left\{\mathbf{y} \in \mathbb{H}^{s}(\Omega) \mid \operatorname{div} \mathbf{y}=0, \mathbf{y} \cdot \vec{n}=0 \text { on } \Gamma\right\}, s \geq 0, \\
V_{0}^{s} & :=V_{n}^{s}, s \in\left[0, \frac{1}{2}\right), \\
V_{0}^{s} & :=\left\{\mathbf{y} \in \mathbb{H}^{s}(\Omega) \mid \operatorname{div} \mathbf{y}=0, \mathbf{y}=0 \text { on } \Gamma\right\}, s>\frac{1}{2} .
\end{aligned}
$$

Moreover, we consider $A_{\alpha}:=A-\alpha I$ where $A$ is the Stokes-Oseen operator and $\alpha$ is such that $A_{\alpha}$ generates an exponentially stable and contractive semigroup on $Y$. From 
[5, Theorem 20], let us recall that

$$
\mathcal{D}\left(\left(-A_{\alpha}^{*}\right)^{\theta}\right)=\left[V_{0}^{2}, Y\right]_{1-\theta}=V_{0}^{2 \theta}, \quad \forall \theta \in[0,1], \quad \theta \neq \frac{1}{4}
$$

While not needed for our purposes, let us emphasize that the case $\theta=\frac{1}{4}$ is also included in [5, Theorem 20].

Using the above notation, for $\varepsilon \in\left(0, \frac{1}{2}\right)$ let us consider the space

$$
W_{\infty}\left(V_{0}^{1+\varepsilon},\left(V_{0}^{1-\varepsilon}\right)^{\prime}\right):=\left\{\mathbf{y} \in L^{2}\left(0, \infty ; V_{0}^{1+\varepsilon}\right) \mid \frac{\mathrm{d}}{\mathrm{d} t} \mathbf{y} \in L^{2}\left(0, \infty ;\left(V_{0}^{1-\varepsilon}\right)^{\prime}\right)\right\}
$$

As mentioned in, e.g., [4], it holds that

$$
\left[V_{0}^{1+\varepsilon},\left(V_{0}^{1-\varepsilon}\right)^{\prime}\right]_{\frac{1}{2}}=\left[\mathcal{D}\left(\left(-A_{\alpha}^{*}\right)^{\frac{1+\varepsilon}{2}}\right), \mathcal{D}\left(\left(-A_{\alpha}^{*}\right)^{\frac{\varepsilon-1}{2}}\right)\right]_{\frac{1}{2}}=\mathcal{D}\left(\left(-A_{\alpha}^{*}\right)^{\frac{\varepsilon}{2}}\right)=V_{0}^{\varepsilon}
$$

From [34, Theorem 4.2], we thus conclude that

$$
W_{\infty}\left(V_{0}^{1+\varepsilon},\left(V_{0}^{1-\varepsilon}\right)^{\prime}\right) \hookrightarrow C_{b}\left([0, \infty) ; V_{0}^{\varepsilon}\right)
$$

where $C_{b}$ denotes the space of continuous and bounded functions.

Before we continue, let us cite the following result from [26].

Proposition 25 [26, B.1] Let $\lambda, \mu, \omega \in \mathbb{R}$. One has for $f \in H^{\lambda+\mu}(\Omega)$ and $g \in$ $H^{\lambda+\omega}(\Omega)$ (where $\Omega$ is a smooth open subset of $\mathbb{R}^{n}$ ):

$$
\|f g\|_{H^{\lambda}(\Omega)} \leq C\|f\|_{H^{\lambda+\mu}(\Omega)}\|g\|_{H^{\lambda+\omega}(\Omega)},
$$

provided that

(i) $\mu+\omega+\lambda \geq \frac{n}{2}$, and

(ii) $\mu \geq 0, \omega \geq 0,2 \lambda \geq-\mu-\omega$, and

(iii) $\mu+\omega+\lambda>\frac{n}{2}$ if equality holds somewhere in (ii).

These estimates allow us to bound the coupling terms appearing in the adjoint equation.

Lemma 26 Let $\varepsilon \in\left(0, \frac{1}{2}\right)$. Let $\overline{\mathbf{y}} \in W_{\infty}$ and $\mathbf{p} \in W_{\infty}\left(V_{0}^{1+\varepsilon},\left(V_{0}^{1-\varepsilon}\right)^{\prime}\right)$. Then

$$
\begin{aligned}
\left\|(\nabla \overline{\mathbf{y}})^{T} \mathbf{p}\right\|_{L^{2}\left(0, \infty ;\left(V_{0}^{1-\varepsilon}\right)^{\prime}\right)} & \leq M_{1}\|\overline{\mathbf{y}}\|_{L^{2}(0, \infty ; V)}\|\mathbf{p}\|_{W_{\infty}\left(V_{0}^{1+\varepsilon},\left(V_{0}^{1-\varepsilon}\right)^{\prime}\right)} \\
\|(\overline{\mathbf{y}} \cdot \nabla) \mathbf{p}\|_{L^{2}\left(0, \infty ;\left(V_{0}^{1-\varepsilon}\right)^{\prime}\right)} & \leq M_{2}\|\overline{\mathbf{y}}\|_{L^{\infty}(0, \infty ; Y)}\|\mathbf{p}\|_{W_{\infty}\left(V_{0}^{1+\varepsilon},\left(V_{0}^{1-\varepsilon}\right)^{\prime}\right)} .
\end{aligned}
$$


Proof For the first assertion, consider $\mathbf{p}_{i} \frac{\partial \overline{\mathbf{y}}_{j}}{\partial x_{k}}$ with $i, j, k \in\{1,2\}$. Set $\lambda=\varepsilon-1, \mu=$ $1, \omega=1-\varepsilon$. Then

$$
\begin{array}{r}
\mu+\omega+\lambda=1+(1-\varepsilon)+(\varepsilon-1)=1 \geq \frac{n}{2}, \\
\mu>0, \omega>0,2 \lambda+\mu+\omega=2(\varepsilon-1)+1+(1-\varepsilon)=\varepsilon>0 .
\end{array}
$$

Applying Proposition 25 with $f=\mathbf{p}_{i}$ and $g=\frac{\partial \overline{\mathbf{y}}_{j}}{\partial x_{k}}$ yields

$$
\int_{0}^{\infty}\|f g\|_{H^{\varepsilon-1}(\Omega)}^{2} \mathrm{~d} t \leq M \int_{0}^{\infty}\left\|\mathbf{p}_{i}\right\|_{H^{\varepsilon}(\Omega)}^{2}\left\|\frac{\partial \overline{\mathbf{y}}_{j}}{\partial x_{k}}\right\|_{L^{2}(\Omega)}^{2} \mathrm{~d} t
$$

which shows the first statement. For the second statement, set $f=\mathbf{y}_{i}, g=\frac{\partial \mathbf{p}_{j}}{\partial x_{k}}, \lambda=$ $\varepsilon-1, \mu=1-\varepsilon$ and $\omega=1$.

The following lemma is formulated for an abstract generator $\widetilde{A}$ of an analytic exponentially stable semigroup on $Y$. It will subsequently be applied with $\widetilde{A}=A_{\alpha}$.

Lemma 27 Let $\tilde{A} \in \mathcal{L}\left(V, V^{\prime}\right)$ generate an exponentially stable semigroup on $Y$ and assume that there exists $M \geq 0$ such that for every $\mathbf{f} \in L^{2}\left(0, \infty ; V^{\prime}\right)$ there exists a unique $\mathbf{y} \in W_{\infty}$ satisfying

$$
\dot{\mathbf{y}}=\widetilde{A} \mathbf{y}+\mathbf{f} \text { on }[0, \infty), \quad \mathbf{y}(0)=0, \quad\|\mathbf{y}\|_{W_{\infty}} \leq M\|\mathbf{f}\|_{L^{2}\left(0, \infty ; V^{\prime}\right)}
$$

Then there exists $\tilde{M}$ such that for all $\Phi \in L^{2}\left(0, \infty ; V^{\prime}\right)$ there exists a unique $\mathbf{r} \in W_{\infty}$ such that

$$
-\dot{\mathbf{r}}=\tilde{A}^{*} \mathbf{r}+\Phi, \quad\|\mathbf{r}\|_{W_{\infty}} \leq \tilde{M}\|\Phi\|_{L^{2}\left(0, \infty ; V^{\prime}\right)}
$$

Proof Step 1. Let us define $T: W_{\infty}^{0} \rightarrow L^{2}\left(0, \infty ; V^{\prime}\right)$ by $T \mathbf{y}=\dot{\mathbf{y}}-\tilde{A} \mathbf{y}$. Considering the adjoint $T^{*}: L^{2}(0, \infty ; V) \rightarrow\left(W_{\infty}^{0}\right)^{\prime}$ we have:

$$
\begin{aligned}
\left\langle T^{*} \boldsymbol{\varphi}, \mathbf{y}\right\rangle_{\left(W_{\infty}^{0}\right)^{\prime}, W_{\infty}^{0}} & :=\langle\boldsymbol{\varphi}, T \mathbf{y}\rangle_{L^{2}(0, \infty ; V), L^{2}\left(0, \infty ; V^{\prime}\right)} \\
& =\langle\boldsymbol{\varphi}, \dot{\mathbf{y}}-\widetilde{A} \mathbf{y}\rangle_{L^{2}(0, \infty ; V), L^{2}\left(0, \infty ; V^{\prime}\right)}
\end{aligned}
$$

Since, by assumption, $T$ is a homeomorphism it is in particular surjective and injective and by the closed range theorem there exists a constant $C$ such that

$$
\|\varphi\|_{L^{2}(0, \infty ; V)} \leq C\left\|T^{*} \varphi\right\|_{\left(W_{\infty}^{0}\right)^{\prime}}, \quad \forall \varphi \in L^{2}(0, \infty ; V)
$$

Step 2. Let $\boldsymbol{\Phi} \in L^{2}\left(0, \infty ; V^{\prime}\right)$ be arbitrary. Then there exists a unique $\mathbf{r} \in L^{2}(0, \infty ; V)$ such that $T^{*} \mathbf{r}=\boldsymbol{\Phi}$, and by (70) we have $\|\mathbf{r}\|_{L^{2}(0, \infty ; V)} \leq C\|\boldsymbol{\Phi}\|_{\left(W_{\infty}^{0}\right)^{\prime}} \leq$ 
$C\|\boldsymbol{\Phi}\|_{L^{2}\left(0, \infty ; V^{\prime}\right)}$. Since $T^{*} \mathbf{r}=\boldsymbol{\Phi}$ we have for all $\mathbf{y} \in W_{\infty}^{0}$

$$
\begin{aligned}
\langle\boldsymbol{\Phi}, \mathbf{y}\rangle_{L^{2}\left(0, \infty ; V^{\prime}\right), L^{2}(0, \infty ; V)}= & \langle\mathbf{r}, T \mathbf{y}\rangle_{L^{2}(0, \infty ; V), L^{2}\left(0, \infty ; V^{\prime}\right)} \\
= & \langle\mathbf{r}, \dot{\mathbf{y}}\rangle_{L^{2}(0, \infty ; V), L^{2}\left(0, \infty ; V^{\prime}\right)} \\
& -\left\langle\widetilde{A}^{*} \mathbf{r}, \mathbf{y}\right\rangle_{L^{2}\left(0, \infty ; V^{\prime}\right), L^{2}(0, \infty ; V)}
\end{aligned}
$$

This implies that the time derivative of $\mathbf{r}$, in the sense of distributions, can be extended to a linear form on $W_{\infty}^{0}$ with the formula:

$$
\begin{aligned}
\langle\dot{\mathbf{r}}, \mathbf{y}\rangle_{\left(W_{\infty}^{0}\right)^{\prime}, W_{\infty}^{0}} & =-\langle\mathbf{r}, \dot{\mathbf{y}}\rangle_{L^{2}(0, \infty ; V), L^{2}\left(0, \infty ; V^{\prime}\right)} \\
& =-\left\langle\boldsymbol{\Phi}+\widetilde{A}^{*} \mathbf{r}, \mathbf{y}\right\rangle_{L^{2}\left(0, \infty ; V^{\prime}\right), L^{2}(0, \infty ; V)}, \forall \mathbf{y} \in W_{\infty}^{0}
\end{aligned}
$$

We estimate

$$
\begin{aligned}
\left\langle\boldsymbol{\Phi}+\tilde{A}^{*} \mathbf{r}, \mathbf{y}\right\rangle_{L^{2}\left(0, \infty ; V^{\prime}\right), L^{2}(0, \infty ; V)} \leq & \left\|\boldsymbol{\Phi}+\widetilde{A}^{*} \mathbf{r}\right\|_{L^{2}(0, \infty ; V)}\|\mathbf{y}\|_{L^{2}(0, \infty ; V)} \\
\leq & \|\boldsymbol{\Phi}\|_{L^{2}\left(0, \infty ; V^{\prime}\right)}\|\mathbf{y}\|_{L^{2}(0, \infty ; V)} \\
& +C\|\boldsymbol{\Phi}\|_{L^{2}\left(0, \infty ; V^{\prime}\right)}\|\mathbf{y}\|_{L^{2}(0, \infty ; V)} \\
= & (1+C)\|\boldsymbol{\Phi}\|_{L^{2}\left(0, \infty ; V^{\prime}\right)}\|\mathbf{y}\|_{L^{2}(0, \infty ; V)}
\end{aligned}
$$

Together with (71) and recalling that $W_{\infty}^{0}$ is dense in $L^{2}(0, \infty ; V)$, we obtain that $\dot{\mathbf{r}}$ can be extended to a bounded linear form on $L^{2}(0, \infty ; V)$, i.e., $\dot{\mathbf{r}}$ can be extended to an element of $L^{2}\left(0, \infty ; V^{\prime}\right)$, moreover,

$$
\|\dot{\mathbf{r}}\|_{L^{2}\left(0, \infty ; V^{\prime}\right)} \leq(1+C)\|\boldsymbol{\Phi}\|_{L^{2}\left(0, \infty ; V^{\prime}\right)}
$$

It follows that $\mathbf{r} \in W_{\infty}$. Moreover,

$$
\|\mathbf{r}\|_{W_{\infty}} \leq 2(1+C)\|\boldsymbol{\Phi}\|_{L^{2}\left(0, \infty ; V^{\prime}\right)} \text { and }-\dot{\mathbf{r}}-\widetilde{A}^{*} \mathbf{r}=\boldsymbol{\Phi} \text { in } L^{2}\left(0, \infty ; V^{\prime}\right)
$$

Corollary 28 Let $\varepsilon \in\left(0, \frac{1}{2}\right)$. For all $\boldsymbol{\Phi} \in L^{2}\left(0, \infty ;\left(V_{0}^{1-\varepsilon}\right)^{\prime}\right)$, the system

$$
-\dot{\mathbf{r}}=A_{\alpha}^{*} \mathbf{r}+\boldsymbol{\Phi}
$$

has a unique solution $\mathbf{r} \in W_{\infty}\left(V_{0}^{1+\varepsilon},\left(V_{0}^{1-\varepsilon}\right)^{\prime}\right)$. Moreover, there exists a constant $M_{\alpha}>0$ independent of $\boldsymbol{\Phi}$ such that

$$
\|\mathbf{r}\|_{W_{\infty}\left(V_{0}^{1+\varepsilon},\left(V_{0}^{1-\varepsilon}\right)^{\prime}\right)} \leq M_{\alpha}\|\boldsymbol{\Phi}\|_{L^{2}\left(0, \infty ;\left(V_{0}^{1-\varepsilon}\right)^{\prime}\right)} \cdot
$$

Proof We appy Lemma 27 to $\dot{\mathbf{z}}=A_{\alpha}^{*} \mathbf{z}+\boldsymbol{\Psi}$ with $\boldsymbol{\Psi}=\left(-A_{\alpha}^{*}\right)^{\frac{\varepsilon}{2}} \boldsymbol{\Phi} \in L^{2}\left(0, T ; V^{\prime}\right)$, to obtain $\|\mathbf{z}\|_{W_{\infty}} \leq \tilde{M}\|\boldsymbol{\Phi}\|_{L^{2}\left(0, \infty ; V^{\prime}\right)}$. Setting $\mathbf{r}:=\left(-A_{\alpha}^{*}\right)^{-\frac{\varepsilon}{2}} \mathbf{z}$ and using that $\left(-A_{\alpha}^{*}\right)^{-\frac{\varepsilon}{2}}$ 
is an isomorphism from $V$ to $V_{0}^{1+\varepsilon}$ and from $V^{\prime}$ to $\left(V_{0}^{1-\varepsilon}\right)^{\prime}$, [41, Section 1.15.2, p. 101], the claim follows.

Proof of Proposition 15 Only regularity has to be shown. Let us fix $\varepsilon \in\left(0, \frac{1}{2}\right)$ and let $M_{\alpha}$ be given by Corollary 28.

Let us define

$$
\begin{aligned}
\mathcal{M} & :=\left\{\mathbf{q} \in W_{\infty}\left(V_{0}^{1+\varepsilon},\left(V_{0}^{1-\varepsilon}\right)^{\prime}\right) \mid\|\mathbf{q}\|_{W_{\infty}\left(V_{0}^{1+\varepsilon},\left(V_{0}^{1-\varepsilon}\right)^{\prime}\right)} \leq 2 M_{\alpha} \gamma\right\}, \\
\gamma & :=\alpha\|\mathbf{p}\|_{L^{2}(0, \infty ; V)}+\|\overline{\mathbf{y}}\|_{L^{2}(0, \infty ; V)} .
\end{aligned}
$$

Let us then choose $\tilde{\delta}_{4}>0$ such that

$$
\|\overline{\mathbf{y}}\|_{L^{2}(0, \infty ; V)} \leq M\left\|\mathbf{y}_{0}\right\|_{Y} \leq M \tilde{\delta}_{4} \leq \frac{1}{2\left(M_{1}+M_{2}\right) M_{\alpha}},
$$

where $M_{1}$ and $M_{2}$ are given by Lemma 26 . Further consider the mapping $\mathcal{Z}$ defined by

$$
\mathcal{Z}: \mathcal{M} \ni \mathbf{q} \mapsto \mathbf{r} \in W_{\infty}\left(V_{0}^{1+\varepsilon},\left(V_{0}^{1-\varepsilon}\right)^{\prime}\right)
$$

where $\mathbf{r}$ is the unique solution of

$$
-\dot{\mathbf{r}}=A_{\alpha}^{*} \mathbf{r}+(\overline{\mathbf{y}} \cdot \nabla) \mathbf{q}-(\nabla \overline{\mathbf{y}})^{T} \mathbf{q}+\alpha \mathbf{p}+\overline{\mathbf{y}}
$$

according to Lemma 26 and Corollary 28. Given $\mathbf{q} \in \mathcal{M}$, it holds that

$$
\begin{aligned}
\|\mathbf{r}\|_{W_{\infty}\left(V_{0}^{1+\varepsilon},\left(V_{0}^{1-\varepsilon}\right)^{\prime}\right)} & \leq M_{\alpha}\left(\left\|(\overline{\mathbf{y}} \cdot \nabla) \mathbf{q}-(\nabla \overline{\mathbf{y}})^{T} \mathbf{q}+\alpha \mathbf{p}+\overline{\mathbf{y}}\right\|_{L^{2}\left(0, \infty ;\left(V_{0}^{1-\varepsilon}\right)^{\prime}\right)}\right) \\
& \leq M_{\alpha}\left(\left(M_{1}+M_{2}\right)\|\overline{\mathbf{y}}\|_{L^{2}(0, \infty ; V)}\|\mathbf{q}\|_{W_{\infty}\left(V_{0}^{1+\varepsilon},\left(V_{0}^{1-\varepsilon}\right)^{\prime}\right)}+\gamma\right) \\
& \leq M_{\alpha}\left(\frac{1}{2 M_{\alpha}}\|\mathbf{q}\|_{W_{\infty}\left(V_{0}^{1+\varepsilon},\left(V_{0}^{1-\varepsilon}\right)^{\prime}\right)}+\gamma\right) \leq 2 M_{\alpha} \gamma .
\end{aligned}
$$

We obtain that $\mathcal{Z}(\mathcal{M}) \subseteq \mathcal{M}$. Consider $\mathbf{q}_{1}, \mathbf{q}_{2} \in \mathcal{Z}$ and let $\mathbf{r}=\mathcal{Z}\left(\mathbf{q}_{1}\right)-\mathcal{Z}\left(\mathbf{q}_{2}\right)$. Note that $\mathbf{r}$ solves

$$
-\dot{\mathbf{r}}=A_{\alpha}^{*} \mathbf{r}+(\overline{\mathbf{y}} \cdot \nabla)\left(\mathbf{q}_{1}-\mathbf{q}_{2}\right)-(\nabla \overline{\mathbf{y}})^{T}\left(\mathbf{q}_{1}-\mathbf{q}_{2}\right)
$$

so that we obtain

$$
\begin{aligned}
\left\|\mathcal{Z}\left(\mathbf{q}_{1}\right)-\mathcal{Z}\left(\mathbf{q}_{2}\right)\right\|_{W_{\infty}\left(V_{0}^{1+\varepsilon},\left(V_{0}^{1-\varepsilon}\right)^{\prime}\right)} & =\|\mathbf{r}\|_{W_{\infty}\left(V_{0}^{1+\varepsilon},\left(V_{0}^{1-\varepsilon}\right)^{\prime}\right)} \\
& \leq M_{\alpha}\left(M_{1}+M_{2}\right)\|\overline{\mathbf{y}}\|_{W_{\infty}}\left\|\mathbf{q}_{1}-\mathbf{q}_{2}\right\|_{W_{\infty}\left(V_{0}^{1+\varepsilon},\left(V_{0}^{1-\varepsilon}\right)^{\prime}\right)} \\
& \leq \frac{1}{2}\left\|\mathbf{q}_{1}-\mathbf{q}_{2}\right\|_{W_{\infty}\left(V_{0}^{1+\varepsilon},\left(V_{0}^{1-\varepsilon}\right)^{\prime}\right)} .
\end{aligned}
$$


Thus by the Banach fixed point theorem we conclude that there exists $\mathbf{r} \in$ $W_{\infty}\left(V_{0}^{1+\varepsilon},\left(V_{0}^{1-\varepsilon}\right)^{\prime}\right) \subset W_{\infty}$ which is a solution of

$$
-\dot{\mathbf{r}}=A_{\alpha}^{*} \mathbf{r}+(\overline{\mathbf{y}} \cdot \nabla) \mathbf{r}-(\nabla \overline{\mathbf{y}})^{T} \mathbf{r}+\alpha \mathbf{p}+\mathbf{N}
$$

It remains to show that $\mathbf{r}$ solves (25). For this, we define $\mathbf{e}:=\mathbf{r}-\mathbf{p} \in L^{2}(0, \infty ; V)$ and observe that e satisfies

$$
T^{*} \mathbf{e}=G^{*} \mathbf{e} \text { in }\left(W_{\infty}^{0}\right)^{\prime}
$$

where the operator $G \in \mathcal{L}\left(W_{\infty}, L^{2}\left(0, \infty ; V^{\prime}\right)\right)$ is defined in (30). It follows from (70) that

$$
\|\mathbf{e}\|_{L^{2}(0, \infty ; V)} \leq M\left\|T^{*} \mathbf{e}\right\|_{\left(W_{\infty}^{0}\right)^{\prime}} \leq M\left\|G^{*}\right\|_{\mathcal{L}\left(L^{2}(0, \infty ; V),\left(W_{\infty}\right)^{\prime}\right)}\|\mathbf{e}\|_{L^{2}(0, \infty ; V)} .
$$

As a consequence of (29), $\tilde{\delta}_{4}$ can be reduced so that $\left\|G^{*}\right\|=\|G\|<\frac{1}{M}$. Hence, we obtain $\mathbf{e}=0$ and thus $\mathbf{r}=\mathbf{p}$ showing that $\mathbf{p} \in W_{\infty}\left(V_{0}^{1+\varepsilon},\left(V_{0}^{1-\varepsilon}\right)^{\prime}\right) \subset W_{\infty}$.

\section{References}

1. Abergel, F., Temam, R.: On some control problems in fluid mechanics. Theor. Comput. Fluid Dyn. 1, 303-325 (1990)

2. Aguilar, C.O., Krener, A.J.: Numerical solutions to the Bellman equation of optimal control. J. Optim. Theory Appl. 160, 527-552 (2014)

3. Al'brekht, E.: On the optimal stabilization of nonlinear systems. J. Appl. Math. Mech. 25, 1254-1266 (1961)

4. Badra, M.: Abstract settings for stabilization of nonlinear parabolic system with a Riccati-based strategy. Application to Navier-Stokes and Boussinesq equations with Neumann or Dirichlet control. Discret. Contin. Dyn. Syst. A 32, 1169-1208 (2012)

5. Badra, M., Takahashi, T.: Stabilization of parabolic nonlinear systems with finite dimensional feedback or dynamical controllers: application to the Navier-Stokes system. SIAM J. Control Optim. 49, 420463 (2011)

6. Barbu, V.: Stabilization of Navier-Stokes Flows. Communications and Control Engineering Series. Springer, London (2011)

7. Barbu, V., Lasiecka, I., Triggiani, R.: Tangential boundary stabilization of Navier-Stokes equations. Mem. Am. Math. Soc. 181, 1-128 (2006)

8. Beeler, S., Tran, H., Banks, H.: Feedback control methodologies for nonlinear systems. J. Optim. Theory Appl. 107, 1-33 (2000)

9. Behr, M., Benner, P., Heiland, J.: Example setups of Navier-Stokes equations with control and observation: spatial discretization and representation via linear-quadratic matrix coefficients. Technical Report, Max Planck Institute for Complex Dynamical Systems. arXiv:1707.08711 (2017)

10. Benner, P., Heiland, J.: Robust stabilization of laminar flows in varying flow regimes. IFACPapersOnLine. In: 2nd IFAC Workshop on Control of Systems Governed by Partial Differential Equations CPDE 2016, 49, pp. 31-36 (2016)

11. Benner, P., Saak, J., Stoll, M., Weichelt, H.: Efficient solution of large-scale saddle point systems arising in Riccati-based boundary feedback stabilization of incompressible Stokes flow. SIAM J. Sci. Comput. 35, S150-S170 (2013)

12. Bensoussan, A., Da Prato, G., Delfour, M., Mitter, S.: Representation and Control of Infinite Dimensional Systems. Birkhäuser, Boston, Basel, Berlin (2007)

13. Bewley, T., Temam, R., Ziane, M.: Existence and uniqueness of optimal control to the Navier-Stokes equations. C. R. l'Acad. Sci. Sér. I 330, 1007-1011 (2000) 
14. Boyer, F., Fabrie, P.: Mathematical Tools for the Study of the Incompressible Navier-Stokes Equations and Related Models. Applied Mathematical Sciences, vol. 183. Springer, New York (2013)

15. Breiten, T., Kunisch, K., Pfeiffer, L.: Infinite-horizon bilinear optimal control problems: sensitivity analysis and polynomial feedback laws. SIAM J. Control Optim. 56, 3184-3214 (2018)

16. Breiten, T., Kunisch, K., Pfeiffer, L.: Numerical study of polynomial feedback laws for a bilinear control problem. Math. Control Relat. Fields 8, 557-582 (2018)

17. Breiten, T., Kunisch, K., Pfeiffer, L.: Taylor expansions of the value function associated with a bilinear optimal control problem. Ann l'Inst. Henri Poincaré C, Anal. Non Linéaire. Accepted for publication (2019)

18. Casas, E.: An optimal control problem governed by the evolution Navier-Stokes equations. In: Optimal Control of Viscous Flow. SIAM, Philadelphia, PA, pp. 79-95 (1998)

19. Collis, S.S., Ghayour, K., Heinkenschloss, M., Ulbrich, M., Ulbrich, S.: Numerical Solution of Optimal Control Problems Governed by the Compressible Navier-Stokes Equations, in Optimal Control of Complex Structures (Oberwolfach, 2000). International Series of Numerical Mathematics, vol. 139, pp. 43-55. Birkhäuser, Basel (2002)

20. Curtain, R.F., Zwart, H.J.: An Introduction to Infinite-Dimensional Linear Systems Theory. Springer, New York (2005)

21. De Los Reyes, J.C., Griesse, R.: State-constrained optimal control of the three-dimensional stationary Navier-Stokes equations. J. Math. Anal. Appl. 343, 257-272 (2008)

22. Desai, M., Ito, K.: Optimal controls of Navier-Stokes equations. SIAM J. Control Optim. 32, 14281446 (1994)

23. Fernández-Cara, E., Guerrero, S., Imanuvilov, O.Y., Puel, J.-P.: Local exact controllability of the Navier-Stokes system. J. Mat. Pures Appl. Neuvième Série 83, 1501-1542 (2004)

24. Fursikov, A.V.: Stabilizability of two-dimensional Navier-Stokes equations with help of a boundary feedback control. J. Math. Fluid Mech. 3, 259-301 (2001)

25. Grasedyck, L.: Existence and computation of low Kronecker-rank approximations for large linear systems of tensor product structure. Computing 72, 247-265 (2004)

26. Grubb, G., Solonnikov, V.: Boundary value problems for the nonstationary Navier-Stokes equations treated by pseudo-differential methods. Math. Scand. 69, 217-290 (1991)

27. Gunzburger, M.D., Hou, L., Svobodny, T.P.: Analysis and finite element approximation of optimal control problems for the stationary Navier-Stokes equations with distributed and Neumann controls. Math. Comput. 57, 123-151 (1991)

28. Heinkenschloss, M., Sorensen, D., Sun, K.: Balanced truncation model reduction for a class of descriptor systems with application to the Oseen equations. SIAM J. Sci. Comput. 30, 1038-1063 (2008)

29. Hinze, M.: Optimal and Instantaneous Control of the Instationary Navier-Stokes Equations, Habilitation Thesis, Technical University, Berlin (2000)

30. Hinze, M., Kunisch, K.: Second order methods for optimal control of time-dependent fluid flow. SIAM J. Control Optim. 40, 925-946 (2001)

31. Ito, K., Ravindran, S.S.: Optimal control of thermally convected fluid flows. SIAM J. Sci. Comput. 19, 1847-1869 (1998)

32. Kato, T.: Perturbation Theory for Linear Operators. Springer, Berlin/Heidelberg (1980)

33. Lasiecka, I., Triggiani, R.: Control Theory for Partial Differential Equations. Abstract Parabolic Systems: Continuous and Approximation Theories, vol. 1. Cambridge University Press, Cambridge (2000)

34. Lions, J.-L., Magenes, E.: Non-homogeneous Boundary Value Problems and Applications. Die Grundlehren der Mathematischen Wissenschaften in Einzeldarstellungen, vol. I. Springer, Berlin (1972)

35. Lukes, D.L.: Optimal regulation of nonlinear dynamical systems. SIAM J. Control 7, 75-100 (1969)

36. Navasca, C., Krener, A.: Patchy Solutions of Hamilton-Jacobi-Bellman Partial Differential Equations, pp. 251-270. Springer, Berlin, Heidelberg (2007)

37. Pritchard, A.J., Zabczyk, J.: Stability and stabilizability of infinite-dimensional systems. SIAM Rev. 23, 25-52 (1981)

38. Raymond, J.-P.: Feedback boundary stabilization of the two-dimensional Navier-Stokes equations. SIAM J. Control Optim. 45, 790-828 (2006)

39. Simon, J.: On the existence of the pressure for solutions of the variational Navier-Stokes equations. J. Math. Fluid Mech. 1, 225-234 (1999)

40. Temam, R.: Navier-Stokes Equations. Studies in Mathematics and its Applications, vol. 2, Revised edn. North-Holland Publishing Co., Amsterdam, New York (1979) 
41. Triebel, H.: Interpolation Theory, Function Spaces, Differential Operators. North-Holland Publishing Company, Amsterdam (1978)

42. Tröltzsch, F., Wachsmuth, D.: Second-order sufficient optimality conditions for the optimal control of Navier-Stokes equations. ESAIM Control Optim. Calc. Var. 12, 93-119 (2006)

43. Zeidler, E.: Nonlinear Functional Analysis and its Applications. I. Fixed-Point Theorems. Springer, New York (1986)

Publisher's Note Springer Nature remains neutral with regard to jurisdictional claims in published maps and institutional affiliations. 